OPEN ACCESS

Edited by:

Jiajia Zhu,

First Affiliated Hospital of Anhui

Medical University, China

Reviewed by:

Chuanjun Zhuo,

Tianjin Anding Hospital, China

Xiaoxia Du,

Shanghai University of Sport, China

*Correspondence: Wei Chen

srrcw@zju.edu.cn

Jinsong Tang

tangjinsong@zju.edu.cn

Specialty section:

This article was submitted to

Brain Imaging Methods,

a section of the journal

Frontiers in Neuroscience

Received: 09 August 2021

Accepted: 14 October 2021

Published: 11 November 2021

Citation:

Shao X, Liao Y, Gu L, Chen W and Tang J (2021) The Etiology of Auditory Hallucinations in Schizophrenia: From

Multidimensional Levels.

Front. Neurosci. 15:755870.

doi: 10.3389/fnins.2021.755870

\section{The Etiology of Auditory Hallucinations in Schizophrenia: From Multidimensional Levels}

\author{
Xu Shao', Yanhui Liao', Lin Gu ${ }^{2,3}$, Wei Chen ${ }^{1 *}$ and Jinsong Tang ${ }^{1 *}$ \\ ${ }^{1}$ Department of Psychiatry, Sir Run Run Shaw Hospital, Zhejiang University School of Medicine, Hangzhou, China, ${ }^{2}$ RIKEN \\ AIP, Tokyo, Japan, ${ }^{3}$ Research Center for Advanced Science and Technology, The University of Tokyo, Tokyo, Japan
}

Enormous efforts have been made to unveil the etiology of auditory hallucinations (AHs), and multiple genetic and neural factors have already been shown to have their own roles. Previous studies have shown that AHs in schizophrenia vary from those in other disorders, suggesting that they have unique features and possibly distinguishable mechanisms worthy of further investigation. In this review, we intend to offer a comprehensive summary of current findings related to AHs in schizophrenia from aspects of genetics and transcriptome, neurophysiology (neurometabolic and electroencephalogram studies), and neuroimaging (structural and functional magnetic resonance imaging studies and transcriptome-neuroimaging association study). Main findings include gene polymorphisms, glutamate level change, electroencephalographic alterations, and abnormalities of white matter fasciculi, cortical structure, and cerebral activities, especially in multiple regions, including auditory and language networks. More solid and comparable research is needed to replicate and integrate ongoing findings from multidimensional levels.

Keywords: auditory hallucination, DTI, EEG, fMRI, genetics, schizophrenia

\section{INTRODUCTION}

Auditory hallucinations (AHs) are defined as experiences that without an external stimulus, individuals perceive voices as distinct from their own thoughts, whether the voices are familiar or not (American Psychiatric Association, 2013). With nearly 10\% of lifetime prevalence rate among the general population (Maijer et al., 2018), this debilitating symptom occurs among healthy population, as well as people with various clinical conditions such as psychiatric diseases (including schizophrenia, mood disorders, dissociative disorders, etc.), neurological diseases, and hearing impairment (Laroi et al., 2012). AHs are most commonly found in major psychotic disorders, with the lifetime prevalence rate of $60-80 \%$ in schizophrenia spectrum disorders (Lim et al., 2016), and 1-year prevalence rate of 50-70\% in schizophrenia specifically (Bauer et al., 2011; Waters et al., 2014). AHs are a main positive symptom of schizophrenia (American Psychiatric Association, 2013) and can bring severe damage to one's mental health, for instance, increasing depressive symptoms (Chiang et al., 2018) and leading to suicidal ideation or attempt (Koyanagi et al., 2015).

As to the etiology, the past decades have witnessed a rapid growth in clinical studies investigating the genetic and neural substrates for AHs generally and the verbal type specifically [auditory verbal hallucinations (AVHs)] in schizophrenia. Notwithstanding, the possible mechanism remains 
unclear, and the existing findings are divergent to some extent. A comprehensive analysis of the ongoing studies will help depict a clearer picture of what current science knows about AHs in schizophrenia. Thus, in this review, we summarize the results from previous research, especially in the field of genetics, neurobiology, and neuroimaging. And we mainly focus on "trait" studies conducted in schizophrenia, that is, schizophrenia with auditory (verbal) hallucinations were compared to patients without the symptom instead of healthy controls. Another type of research, "state" studies that compare on-the-state period to offthe-state period within the same patient group, is also included in the respective sections. At the end of this review, we further discuss the limitations of previous studies and propose several suggestions for ways forward.

\section{GENETIC RESEARCH}

\section{Genetic Factor}

It is well-known that gene and its interaction with environment play an important role in the development of psychiatric symptoms or disorders. And researchers have been investigating how genetic and environmental factors are linked to AHs.

Most studies were conducted among schizophrenia patients, considering the frequent occurrence of AHs in this population. Abundant studies showed that DNA variations in the cholecystokinin A receptor (CCK-AR) gene contributed to the formation of schizophrenia and AHs (Wei and Hemmings, 1999; Tachikawa et al., 2001; Sanjuan et al., 2004; Toirac et al., 2007). Schizophrenia carrying glutathione S-transferase (GST) $A 1^{*} B$ allele had more severe AHs than non-B carriers (Spalletta et al., 2012). Rajasekaran et al. (2016) found an association between (human leukocyte antigen-G) HLA-G 14-bp Ins/Ins genotype and lifetime presence of third person AHs, and this association was more significant in males with schizophrenia. The genotype frequency of matrix metalloproteinase 1 (MMP1) single-nucleotide polymorphism (SNP) rs470558 was reported to be associated with AHs, and its A allele frequency was higher in schizophrenia with AHs (SZ-AH) than schizophrenia without AHs (SZ-non-AH) (Kim et al., 2012). Dystrobrevin binding protein 1 (DTNBP1) gene was involved with neurotransmission regulation and neurodevelopment in schizophrenia, and its SNP rs4236167 was found to be associated with AHs generally and third-person and abusive form ones specifically (Cheah et al., 2015). One linkage and association analyses found that D8S1769, located $350 \mathrm{~kb}$ upstream of the $5^{\prime}$ end of the first exon of neuregulin 1 (NRG1) gene, had significant linkage signal for SZ-AH, and the frequency of the $\mathrm{G}$ allele of SNP8NRG241930 was significantly higher in SZ-AH compared to healthy controls (Kim et al., 2006). Previous research on the association between serotonin transporter (5-HTT) gene and AHs has been controversial. 5-HTT gene-linked polymorphic region (5-HTTLPR) is generated by a 44-bp deletion in the promoter site with two principal alleles, short $(s)$ and long $(l)$, respectively (Heils et al., 1996). At first, $l$ allele was found to be associated with the frequency or the severity of hallucinations in schizophrenia (Malhotra et al., 1998). However, Sanjuan et al. (2006a) found that $s$ allele was related to emotional response to AHs in schizophrenia, but not to the AH frequency. In another study, 5-HTTLPR polymorphism was also associated with the emotional response to AHs, specifically the distress caused by the symptom, but only with marginal statistical significance (Rivero et al., 2010).

\section{Genetic x Environmental Factor}

Some other genes have been reported to predispose to AHs through the interaction with environmental factors. For instance, forkhead box P2 gene (FOXP2) was involved in the development of the neural systems mediating speech and language (Liegeois et al., 2003). Patients with abnormal FOXP2 function showed significant underactivation in Broca's area and other languagerelated cortical regions (Liegeois et al., 2003). And investigators found that FOXP2 SNPs were associated with SZ-AH (Sanjuan et al., 2006b), but not directly with the symptom of AHs (Tolosa et al., 2010). Further study showed that SNP rs1456031 interacted with childhood parental emotional abuse to predict AVHs (McCarthy-Jones et al., 2014a). Putting these evidences together, FOXP2 mutation might cause the symptom only in the presence of the environmental factor.

Based on previous findings, multiple genes, along with the influences of psychosocial factors, play their own part in the occurrence of AHs. But it is still unclear whether these genes function independently or they interact with each other, and how much percentage each gene makes up to the pathogenesis of AHs. Among them, DTNBP1 seems to be the most potential gene candidate for AHs. It is a schizophrenia susceptibility gene related to regulation of glutamate level (Tang T. T.-T. et al., 2009), and its mRNA expression is lower in AH-related regions including superior temporal gyrus, hippocampus, and dorsolateral prefrontal cortex (Talbot et al., 2004; Weickert et al., 2004; Tang J. et al., 2009).

Although great joint efforts have been made to analyze data of large-scale genome-wide association studies in the population of schizophrenia to search for regulatory genes, such efforts are not yet made to investigate the genetic connection with AHs. Moreover, previous results are mainly derived according to bioinformatics methodologies, and it remains to be solved from molecular biological level how much influence individuals might receive from these genetic mutations.

\section{Transcriptional Factor}

How gene is expressed in the cerebral area offers a clearer picture of genetic function on human brain. And an increasing amount of studies has investigated differentiated gene expression in specific brain areas using postmortem or database of schizophrenia. ATPase type 13A4 (ATP13A4) gene (verbal and social interaction skills) expression was up-regulated in Broca's area of schizophrenia (Gibbons et al., 2020). Expressions of genes related to cell-cell adhesion, synaptic transmission, and neural excitability were enriched in the prefrontal cortex (Pergola et al., 2019). In dorsolateral prefrontal cortex, to be further, expressions of genes related to regulation of cell survival and growth as well as response to external stimuli (Petralia et al., 2020), related to learning and memory processes (Ohayon et al., 2020), 
and related to immune-related functions (Enwright and Lewis, 2021) were up-regulated, whereas expressions of genes related to mitochondrial function (Enwright and Lewis, 2021); related to neurotransmitter release modulation (Tao et al., 2020); related to cell proliferation, differentiation, and transformation (Huang et al., 2019); and related to neuronal homeostasis and intracellular signaling (Petralia et al., 2020) were down-regulated. Oxytocin receptor (social cognition) mRNA was down-regulated in the temporal cortex (Uhrig et al., 2016), and sodium-dependent MI transporter-1 (SLC5A3) gene (metabolic precursor regulation) expression was up-regulated in the superior temporal cortex (Vawter et al., 2019).

There are scattered reports about gene transcription concerning AHs only using peripheral blood samples. The mRNA level of mitochondrial complex I gene (NDUFV2) was found to be positively correlated with both overall and positive symptoms in the first-episode schizophrenia patients (Akarsu et al., 2014). Complement 4A (C4A) mRNA expression was found to be positively correlated with positive symptoms in schizophrenia, specifically the presence and severity of delusions (Melbourne et al., 2018). Using gene expression profiling from schizophrenia and related disorders, four genes were found to decrease in high hallucinatory state (Fn1, Rhobtb3, Aldh1l1, Mpp3), and three genes were found to increase (Arhgef9, Phlda1, S100a6) (Kurian et al., 2011). Only one study used gene expression profiling of postmortem brain samples from prefrontal cortex and found that plexin B1 (PLXNB1) expression was decreased in SZ-AH compared to SZ-non-AH (GilabertJuan et al., 2015). The different expression of PLXNB1 might be a signature of the hallucinatory endophenotype in schizophrenia.

Therefore, cerebral transcriptomic study of $\mathrm{AHs}$ in schizophrenia is scarce. As the importance is increasingly attached to transcriptomics, this research field leaves much to be exploited.

\section{NEUROPHYSIOLOGICAL RESEARCH}

\section{Neurometabolic Study}

Magnetic resonance spectroscopy (MRS) has been an effective tool to measure cerebral metabolic level of targeted substance and has been used to investigate how certain neurochemicals might affect the occurrence of AHs. For example, as to the measurement of Glx level (composite of glutamate and glutamine), schizophrenia with AVHs (SZ-AVH) had higher Glx level than schizophrenia without AVHs (SZ-non-AVH) in the left lateral prefrontal region (Curcic-Blake et al., 2017), although schizophrenia group generally had lower Glx level than the control group in the temporal and frontal areas (Hugdahl et al., 2015; Curcic-Blake et al., 2017). Besides, AH severity was reported to positively correlate with Glx level both in the frontal and temporal regions (Hugdahl et al., 2015). Glutamate excitatory function is usually balanced by $\gamma$-aminobutyric acid (GABA) inhibitory function (Carlsson et al., 2001). Therefore, the glutamate-GABA excitatory-inhibitory imbalance could lead to the development of AHs (Hugdahl and Sommer, 2018). Further, Hjelmervik et al. (2019) found in a larger sample that Glx level was positively correlated with AVH in the left superior temporal gyrus and negatively in the anterior cingulate cortex, but they failed to find any significant result for GABA level. Consequently, they proposed that compared to Glu-GABA imbalance within regions, Glu-Glu imbalance between regions was more plausible especially in the frontal and temporal regions. Therefore, studies above have shown that glutamatergic metabolites serve as a mediating factor in AHs.

Other neurochemical studies remain scarce. N-acetylaspartate/choline (NAA/Cho) ratio in the right thalamus was lower in SZ-AH, relative to SZ-non-AH or healthy controls (Martinez-Granados et al., 2008). Besides, NAA level was decreased in the hippocampus in schizophrenia during the episode of AHs (Heckers, 2001) and was also reported to be negatively correlated with the duration of positive symptoms (Theberge et al., 2003). Moreover, phospholipids (phosphomonoesters and phosphodiesters) and energy metabolism (adenosine triphosphate, inorganic phosphate, and phosphocreatine) in left superior temporal gyrus were both positively correlated with $\mathrm{AH}$ severity in SZ-AH (Nenadic et al., 2014).

Based on existing findings, the association between glutamate level and AHs is relatively solid, but whether GABA level has a role remains inconclusive. Interregional Glu-Glu imbalance rather than intraregional Glu-GABA imbalance is more suitable at present. Findings of other neurochemical and neurometabolic studies remain scarce. It is worthwhile to finding out in future investigations how chemical substance in the brain and their metabolism influence hallucinatory activities of schizophrenia.

\section{Electroencephalogram Study}

The forward model has been illustrated in many studies and can be applied in the auditory system (Wolpert and Miall, 1996; Heinks-Maldonado et al., 2007). When a sound initiates, the auditory feedback can be predicted by the efference copy of the motor command. This corollary discharge is compared with the actual auditory feedback. If the sound is self-generated, the predicted auditory feedback often matches the actual one; the sensory input is suppressed, leading to a dampened auditory experience. If the sound is externally produced, the predicted auditory feedback often contradicts with the actual one, leading to no suppression of the auditory response. During self-generated vocalizations, neural discharges in a majority of auditory cortical neurons are suppressed, and the suppression precedes the onset of vocalizations (Eliades and Wang, 2003), and this suppression helps distinguish between internally and externally initiated sensations. Event-related potential (ERP) results revealed that in healthy controls, N1 to self-voice feedback was dampened compared to alien voice (Heinks-Maldonado et al., 2007). Phase coherence of prespeech electroencephalogram (EEG) was found to be related to reduced speech-onset N1 potentials, showing prespeech neural synchrony suppressed subsequent responsiveness to self-spoken sound (Ford et al., 2007). However, hallucination predisposition in healthy controls might affect voice discrimination and recognition (Pinheiro et al., 2019). These evidences indicate that people with AHs could not 
recognize self-generated voice correctly, and they displayed improperly high response to it, as the result of which the forward model was damaged.

Schizophrenia is characterized by the disturbance of sensory gating mechanism that filters out extraneous stimuli from meaningful sensory inputs to focus attention (Freedman et al., 1987). And auditory sensory gating is one way of directly measuring auditory perceptual abnormality, and it assesses modulation of incoming auditory information $50 \mathrm{~ms}$ into cortical processing (Thoma et al., 2017). At $50 \mathrm{~ms}$ comes a positive wave, P50 potential, as the largest initial cerebral response to an auditory stimulus (Javitt and Freedman, 2015). Sensory gating deficit has been frequently found in schizophrenia (Potter et al., 2006), but limited studies have explored the relationship between sensory gating deficit and AHs. Smith et al. (2013) demonstrated that AVH severity was positively associated with the extent of P50 sensory gating deficit. Faugere et al. (2016) found that schizophrenia with P50 sensory gating impairment had more severe AVH than without that impairment. For the P50, N100, and P200 components, gating ratios were higher in schizophrenia on the state of AVH than those off the state (Thoma et al., 2017). In one magnetoencephalographic study, gating ratio of the magnetic analog of P50 (P50m) in the left hemisphere was positively associated with AHs in schizophrenia (Hirano et al., 2010). These evidences suggest that sensory gating deficit directly contributed to the formation of AHs as well.

Mismatch negativity (MMN) is another EEG-derived ERP, which functions as a neurophysiological index signaling auditory processing (Naatanen et al., 2007) and reflects detection of the change of stimulus in the environment (Salisbury, 2012). Compared to SZ-non-AH, SZ-AH possessed smaller MMNs to duration deviants (Fisher et al., 2008). In SZ-AH, MMN amplitude to gap deviants was negatively correlated with the duration, loudness, and clarity of AHs (Fisher et al., 2012). Furthermore, reduced MMN amplitude was found to be directly correlated with AHs in schizophrenia with early psychosis (Rudolph et al., 2015). Considering previous studies already reported the association between reduced MMN amplitude and general hallucinatory trait in schizophrenia (Youn et al., 2003; Fisher et al., 2011, 2014; Perrin et al., 2018), it is plausible that deficits of auditory processing and external stimulus detection, indicated by MMN alterations, could contribute to the development of AHs.

Studies have found dysfunctional $\gamma$ frequency $(30-100 \mathrm{~Hz})$ oscillations in AHs. $\gamma$-Band oscillations play a role in selecting neurons, which communicate about sensory inputs, and higher cognitive functions including perceptual organization and language processing (Uhlhaas et al., 2008). The auditory steadystate response (ASSR), as one kind of ERPs that is elicited by temporally modulated auditory stimulation, has been used to study neural synchrony in schizophrenia (O'Donnell et al., 2013). Phase locking factor of the left hemisphere source was correlated with AHs in schizophrenia during ASSR to $40-\mathrm{Hz} \gamma$ frequency stimulation (Spencer et al., 2009). In another study, $40-\mathrm{Hz}$ ASSR was found to be diminished in schizophrenia, and phase synchronization between the primary auditory cortices was positively correlated with AHs (Mulert et al., 2011). In SZ-AVH,
40-Hz EEG activity decreased left-laterally, and global measure of phase locking decreased with stimulation (Koenig et al., 2012). Induced $40-\mathrm{Hz} \gamma$ power in the left hemisphere was correlated with AHs in schizophrenia (Hirano et al., 2015). In another, 80-Hz ASSR-BOLD (blood oxygen-level dependent) signal was positively associated with AHs in acute episode schizophrenia (Kuga et al., 2016). Interestingly, when 40-Hz ASSR was divided as early-latency and late-latency $\gamma$ response, researchers did not find group differences of early or late $\gamma$ activity signatures between SZ-AH and SZ-non-AH (Griskova-Bulanova et al., 2016). Apart from ASSR-related results, correlation dimension in the $\gamma$-band in the right prefrontal cortex was more chaotic in schizophrenia with treatment-refractory AHs than counterparts without (Lee et al., 2008). Therefore, despite that participants recruited and measures reflecting $\gamma$-band oscillations differ in previous studies, results could still suggest that deficit of $\gamma$ oscillations in schizophrenia was related to AHs.

Alterations of other frequency spectrums and prominent spectral interactions are also reported to be associated with AHs. Compared to SZ-non-AH, SZ-AH had increased $\alpha$ band coherence between the left and right superior temporal cortices (Sritharan et al., 2005). Schizophrenia with AHs also had increased $\alpha$-band phase-coupling intrahemispherically and interhemispherically and increased $\alpha$-band synchrony (Angelopoulos et al., 2011). Correlation dimension in the $\beta$-band in the left parietal cortex was more coherent in schizophrenia with treatment-refractory AHs than counterparts without (Lee et al., 2008). Phase coupling between theta and $\gamma$ rhythms was increased in the left frontotemporal cortices during AVH (Koutsoukos et al., 2013). $\beta 1$ and $\beta 2$ frequency amplitude were higher in SZ-AH than SZ-non-AH (Lee et al., 2006). Also, $\gamma$ frequency was correlated with $\beta$ (2 and 3) frequencies in SZ$\mathrm{AH}$, and $\beta$ ( 1 and 2 ) activity was enhanced in the left inferior parietal lobule and the left medial frontal gyrus in SZ-AH relative to SZ-non-AH (Lee et al., 2006).

Combining these evidences together, SZ-AH are deficient in auditory processing, including suppressing inner speech, filtering out meaningless auditory stimuli, detecting stimulus change in the environment, and proper cerebral circuitry function (especially in the primary auditory cortex).

\section{NEUROIMAGING RESEARCH}

\section{Structural Magnetic Resonance Imaging Study \\ Diffusion Tensor Imaging Study}

Compared to SZ-non-AH, SZ-AH had lower fractional anisotropy (FA) in bilateral superior longitudinal fasciculi and arcuate fasciculi (Chawla et al., 2019). Compared to schizophrenia with audiovisual hallucinations, SZ-AH showed lower white matter connectivity in the pathways connecting the hippocampal complex with visual areas including the forceps major and the inferior fronto-occipital fasciculus (Amad et al., 2014). Different correlational studies reported that FAs in bilateral arcuate fasciculus (Rotarska-Jagiela et al., 2009), bilateral 
superior longitudinal fasciculi (Seok et al., 2007; Shergill et al., 2007), and left anterior cingulum (Shergill et al., 2007) were positively associated with AHs in schizophrenia. Meanwhile, mean diffusivity in left superior temporal gyrus white matter was associated with AHs specifically in male schizophrenia (Lee et al., 2009).

Previous studies focusing on verbal type of AHs have yielded inconsistent results. Relative to SZ-non-AVH, SZ-AVH had higher FA in the lateral parts of the temporoparietal section of the arcuate fasciculus (Hubl et al., 2004), in the left arcuate fasciculus (Psomiades et al., 2016), and in parts of the anterior corpus callosum (Hubl et al., 2004). Paradoxically, other studies showed that SZ-AVH had lower FA in the left frontal-temporal regions involved in language networks (including left inferior fronto-occipital fasciculus and left arcuate fasciculus segments) (Curcic-Blake et al., 2015; McCarthy-Jones et al., 2015; Oestreich et al., 2016) and in tracts involved in interhemispheric language connections (including bilateral anterior corona radiata and posterior parts of the corpus callosum) (Curcic-Blake et al., 2015). Mulert et al. (2012) reported higher but Wigand et al. (2015) reported lower FA in the interhemispheric auditory fibers in SZAVH. In correlational analysis, Psomiades et al. (2016) found AVH positively correlated with FA in the left arcuate fasciculus, but Curcic-Blake et al. (2015) found AVH negatively correlated with FA in the left frontal-temporal regions including arcuate fasciculus segments. In addition, some studies did not find the difference of structural integrity of internal capsule (Xi et al., 2016), anterior corona radiata (Xi et al., 2016), the language pathways (Catani et al., 2011; Leroux et al., 2017; Xie et al., 2019), and the interhemispheric auditory pathways (Leroux et al., 2017) between SZ-AVH and SZ-non-AVH, although white matter integrity in SZ-AVH or SZ-non-AVH, respectively, differed from that in healthy controls.

The debate on the involvement of the intrahemispheric and interhemispheric fasciculi in the etiology of AHs thus remains open. And the verbal type might have unique abnormalities of white matter fasciculi relative to the general hallucination. Diffusion tensor imaging (DTI) studies in the past decades are relatively inadequate, and findings of previous studies have been incongruent and contradictory. Still, it can be implied that pathological fasciculus alterations in the language pathways and interhemispheric auditory pathways lead to the emergence of AHs.

\section{Morphological Thickness}

Structural correlates of AHs have been frequently reported in schizophrenia. Compared to SZ-non-AVH, SZ-AVH showed reduced thickness in the right Heschl gyrus (Chen et al., 2015), in the language and primary auditory areas including the Broca's area, the Heschl gyrus, and Wernicke's area of the left hemisphere (van Swam et al., 2012), in the bilateral postcentral gyrus (van Swam et al., 2012), and in the left middle temporal gyrus (Cui et al., 2018). Promisingly, the abnormality of the left Heschl gyrus was replicated in schizophrenia spectrum patients with AHs (Morch-Johnsen et al., 2017), and the abnormality of the left middle temporal gyrus was confirmed by the negative correlation between its cortical thickness and $\mathrm{AH}$ severity (Cui et al., 2018). On the other hand, SZ-AVH showed increased thickness in the frontal cortex (left insular cortex, and bilaterally anterior/posterior cingulate, and dorsal middle frontal gyrus) and parietal lobe (van Swam et al., 2012). Among all, the left middle temporal gyrus is most related to AHs and deserves closer investigation in the future, as it is a vital part of language pathways connected to other areas (Xu et al., 2015) and related to self-monitoring dysfunction (Shergill et al., 2000).

\section{Morphological Volume}

Comparatively, more studies have been conducted concerning structural volume. SZ-AH showed larger volumes of temporal white matter, frontal gray matter, and temporal gray matter when compared to SZ-non-AH (Shin et al., 2005), but Kubera et al. (2014) yielded contrary results when comparing SZ-AVH to schizophrenia without or in remission with AVH. Compared to schizophrenia with audiovisual hallucinations, SZ-AH showed smaller hippocampal complex (Amad et al., 2014). Compared to SZ-non-AVH, SZ-AVH showed increased volume of the right Heschl gyrus (Hubl et al., 2010), but reduced volume of the left insula (Shapleske et al., 2002). Apart from cerebral pathological changes, Cierpka et al. (2017) found that SZ-AVH had lower gray matter volume in lobule VIIIa than SZ-non$\mathrm{AVH}$, suggesting the possible involvement of cerebellum in the pathophysiology of AVH.

There have been studies investigating the direct relationship between the hallucinatory symptom and morphological volume. In a dichotic listening task, $\mathrm{AH}$ severity was negatively correlated with the volume of left anterior superior temporal gyrus in SZ$\mathrm{AH}$ (Levitan et al., 1999). In resting state among schizophrenia, $\mathrm{AH}$ severity was reported to be negatively correlated with the volume of the left superior temporal gyrus (Barta et al., 1990), corpus callosum (Knochel et al., 2012), the left Heschl gyrus, left inferior supramarginal gyrus, right middle/inferior prefrontal gyri (Gaser et al., 2004), the right superior temporal gyrus, right fusiform gyrus, and left inferior temporal gyrus (O'Daly et al., 2007), as well as left inferior frontal gyrus and right postcentral gyrus (Garcia-Marti et al., 2008). On the contrary, other studies found positive correlations between $\mathrm{AH}$ severity and the volume of left inferior frontal gyrus (Modinos et al., 2009), bilateral superior temporal cortex (including Heschl gyrus), left supramarginal/angular gyrus, left postcentral gyrus, and left posterior cingulate cortex (Nenadic et al., 2010). Voxelbased meta-analysis found a negative correlation between $\mathrm{AH}$ severity and gray matter volume in the left insula or right superior temporal gyrus (Palaniyappan et al., 2012). Another meta-analysis found the severity of AVH was correlated with volume reductions in the left and marginally the right superior temporal gyri (including Heschl gyri), implicating the bilateral structural pathology of this region (Modinos et al., 2013). But Modinos et al. (2013) failed to find group difference between SZAVH and SZ-non-AVH. Therefore, correlational findings very much contradict with each other.

Previous structural magnetic resonance imaging (sMRI) studies were mainly conducted in participants' resting state. Evidences have demonstrated morphological changes in certain cerebral regions among schizophrenia, and the majority shows 
the shrinkage rather than the enlargement of the cerebral regions including auditory and language areas. Among all, reduced volume of temporal gyri, including Heschl gyrus, is most frequently replicated in recent research. According to studies of morphological thickness and volume, it is obvious that temporal region plays the most crucial part in the pathogenesis of AHs. However, current MRI results are heterogeneous to a certain extent and are in lack of replicability, which therefore calls for further confirmation of these results in the future.

\section{Functional Magnetic Resonance Imaging Study \\ Cerebral Blood Flow Study}

Studies across decades have shown that cerebral blood flow (CBF) contributes to the neural underpinning of AHs. "State" study showed that schizophrenia had higher blood flow in Broca's area in auditory hallucinating state than in their resolved state (McGuire et al., 1993). Compared to SZ-non-AVH, SZ-AVH displayed increased $\mathrm{CBF}$ in the right superior temporal gyrus and caudate nucleus (Zhuo et al., 2017), in the left superior temporal gyrus and right supramarginal gyrus (Wolf et al., 2012). SZ-AVH also had decreased CBF in the bilateral occipital and left parietal cortices (Zhuo et al., 2017), and in the bilateral superior and middle frontal gyri and postcentral gyri, and right supplementary motor area (Cui et al., 2017a). There were also studies focusing on $\mathrm{CBF}$ during task mode. When generating and monitoring inner speech, SZ-AH had reduced CBF in the left middle temporal gyrus and the rostral supplementary motor area compared to SZ-non-AH (McGuire et al., 1995). During verbal memory activation, SZ-AH had increased $\mathrm{CBF}$ in the left basal ganglia (Busatto et al., 1995). To sum up, CBF studies have implicated increased brain activities among auditory- and language-related regions, which accords with findings in functional MRI (fMRI) studies talked about later. Still, some results are rather sporadic, thus calling for further replications.

\section{Resting-State Functional MRI}

The past decades have witnessed fMRI findings emerging one after another concerning the brain activities during AHs. In order to achieve definite conclusions, meta-analysis has become a powerful systematic tool. Across populations of schizophreniaspectrum disorder, psychotic disorder, and healthy controls, meta-analyses of trait studies revealed that AVHs were frequently related to activations in the left middle and superior temporal gyrus, left postcentral and precentral gyrus, left insula, left hippocampus/parahippocampal region, right inferior frontal gyrus, and so on (Jardri et al., 2011; Kompus et al., 2011; Kühn and Gallinat, 2012; van Lutterveld et al., 2013; Zmigrod et al., 2016). The inferior parietal lobule was also frequently reported in these analyses, but the lateralization was inconsistent. Other less frequent reported regions include anterior cingulate cortex and thalamus. Generally speaking, the cerebral activations in trait studies reflect lateralization in the left hemisphere, and related regions extensively cover the language/speech, auditory, and limbic networks. On the other hand, meta-analysis of state studies revealed that AVHs were associated with activation in bilateral inferior frontal gyrus, bilateral postcentral gyrus, and left parietal operculum (Kühn and Gallinat, 2012).

Functional connectivity is used to detect the temporal correlation of the low-frequency fluctuation in the BOLD signal between regions (Fox and Raichle, 2007), and the abnormalities of interregional resting-state functional connectivity were found among different brain areas including those reported above. Compared to patients without the symptom, schizophrenia spectrum patients with AHs had higher functional connectivity of left Heschl gyrus (belonging to the primary auditory cortex) with left frontoparietal regions and lower one with right hippocampal formation and mediodorsal thalamus, and functional connectivity of the left Heschl gyrus was correlated with $\mathrm{AH}$ severity in regions related to language, memory, and self-monitoring (Shinn et al., 2013). SZ-AH, compared to patients with audiovisual hallucinations, had lower functional connectivity of the bilateral hippocampal complex with the medial prefrontal cortex and the caudate nuclei and had a higher one with the thalamus (Amad et al., 2014), and dysconnectivity of hippocampal subregions was also reported in SZ-AVH (Liu et al., 2019). SZ-AH, compared to SZ-non-AH, displayed enhanced functional connectivity of the bilateral nucleus accumbens with the left superior temporal gyrus, the cingulate gyri, and the ventral tegmental area, indicating that the increased activity of the mesolimbic pathway might underlie the occurrence of AHs (Rolland et al., 2015), while in terms of AVH, SZ-AVH showed higher functional connectivity in a neural circuit involving the anterior cingulate cortex, insula, and language-related areas including superior temporal gyrus and inferior parietal lobule, compared to SZ-non-AVH (Chang et al., 2017). SZ$\mathrm{AVH}$ also showed thalamic-auditory cortical hyperconnectivity and auditory cortical-hippocampal hypoconnectivity, and AVH severity was positively correlated with the connectivity from Broca's area to the auditory cortex (Li et al., 2017). Direct correlational analyses among SZ-AVH showed that AVH severity was negatively correlated with functional connectivity in the left anterior cingulate cortex, positively correlated with the left superior temporal gyrus and right lateral prefrontal cortex (Wolf et al., 2011), and negatively correlated with neural coupling between left temporoparietal junction, bilateral anterior cingulate, and bilateral amygdala (Vercammen et al., 2010).

Within the auditory network, SZ-AH had lower interhemispheric connectivity in both primary and secondary auditory cortices when compared to SZ-non-AH (Gavrilescu et al., 2010). In another study, SZ-AH was reported to have decreased functional connectivity between two regions inside the auditory network, right Heschl gyrus, and right posterior superior temporal gyrus, compared to SZ-non-AH (Guo et al., 2020). Within the language network, SZ-AVH had higher functional connectivity of bilateral Wernicke's area with the left inferior frontal gyrus (Hoffman et al., 2011) and reduced causal interactions from the left inferior frontal gyrus to left middle temporal gyrus (Zhang et al., 2017). In addition, the auditory cortex-posterior language network involving auditory cortex and posterior language regions was more active during AVH-on periods in schizophrenia spectrum disorders, whereas occipital-temporal and medial prefrontal networks were more 
active during AVH-off periods (Thoma et al., 2016). Within the default mode network (DMN), SZ-AVH had lower effective connectivity from anteromedial prefrontal cortex to left inferior temporal gyrus and from posterior cingulate cortex to left cerebellum posterior lobe, inferior temporal gyrus, and right middle frontal gyrus than SZ-non-AVH (Zhao et al., 2018). Although Guo et al. (2020) did not find dysconnectivity in the DMN, they reported that SZ-AH had reduced functional connectivity of overall parietal memory network adjacent to the $\mathrm{DMN}$ and also reduced functional connectivity between core regions, and the latter negatively correlated with $\mathrm{AH}$ severity.

Interactions between multiple networks have also been reported. With independent component analysis and dual regression, Cui et al. (2017b) demonstrated AVH-related coactivation within the auditory, default mode, executive, motor, and frontoparietal networks, which were involved in auditory processing, language production and monitoring, and sensory information filtering. Alonso-Solis et al. (2015) found alterations of resting-state functional connectivity of DMN subsystems with hubs of the salience network, suggesting cross-network abnormalities related to AVH. Further, stochastic dynamic causal modeling analysis captured the link between general ongoing hallucinatory state in schizophrenia with memory-based sensory input from the hippocampus to the salience network (Lefebvre et al., 2016). In another study, Scheinost et al. (2019) put forward a potential AVH network overlying the default mode and language processing networks.

\section{Task-State Functional MRI}

Studies of task-state fMRI related to AHs in schizophrenia are in lack so far. During a voice recognition task, SZ-AVH had reduced functional connectivity of right superior temporal gyrus with right superior frontal gyrus (Mou et al., 2013). Within the inner speech processing network, loudness of AVH in SZAVH was correlated with reduced activity in bilateral angular gyrus, bilateral anterior cingulate gyrus, left inferior frontal gyrus, left insula, and left middle temporal gyrus, during a metrical stress evaluation task activating inner speech production and perception (Vercammen et al., 2011). During verbal speech perception, SZ-AVH displayed hypercoupling in auditory-motor, language processing, and DMNs compared to SZ-non-AVH (Lavigne and Woodward, 2018), and SZ-AH comorbid with other hallucinatory types displayed a hypercoupling left-dominant temporal-frontal network involving speech-related auditory and motor regions (Lavigne et al., 2015).

Currently, neuroimaging results are still insufficient to draw any decisive conclusions. That being said, fMRI has been an effective tool to offer abundant evidences, implying that neural mechanisms underlying AHs involve abnormal activation among multiple cerebral regions related to speech/language processing, auditory perception, and so on, and disordered brain connections can be found at interregional, intranetwork, and internetwork level. Matching sMRI results and abnormal activation of superior and middle temporal gyrus are replicated constantly in fMRI studies, suggesting these regions have both structural and functional abnormalities related to AHs. As to the interregional connection, functional connectivity of Heschl gyrus, superior temporal gyrus, and hippocampus region is most noteworthy. As to intranetwork and internetwork connection, deficits of the auditory network and language network are most prominent, followed by DMN.

\section{Transcriptome-Neuroimaging Study}

Transcriptional and neuroimaging combined studies have received huge popularity in recent years, but few has been conducted related to schizophrenia, let alone AHs. Using postmortem prefrontal cortex samples of male schizophrenia and controls and high-resolution anatomical MRI with optimized voxel-based morphometry, Sanjuán et al. (2021) found low FOXP2 (language mediation) mRNA level was associated with reduced gray matter density, and SNP rs2396753 played a part in this association. One posttranscriptional study using PET imaging to measure the $\alpha 5$ subtype of the GABA receptor $(\alpha 5$ $\mathrm{GABA}_{A} \mathrm{Rs}$ ) availability found that $\alpha 5-\mathrm{GABA}_{A}$ Rs protein level was reduced in the hippocampus of antipsychotic-free schizophrenia and correlated positively with total symptom score (Marques et al., 2020), which extended previous results of lower mRNA and protein levels of $\alpha 5-\mathrm{GABA}_{A}$ Rs in schizophrenia (Duncan et al., 2010; Beneyto et al., 2011). These evidences provided moderate support for central GABA hypofunction underlying the pathophysiology of clinical symptoms, albeit no direct connection with AHs. Only two studies in latest years did belong to transcriptome-neuroimaging association study of AHs. Using high-throughput RNA sequencing and resting-state fMRI data, expression of an lncRNA-mRNA network centered by lncRNA MSTRG.96171.1 was upregulated in SZ-AVH relative to SZ-non$\mathrm{AVH}$, and functional connectivity of DMN regions was positively correlated with AVH severity and MSTRG.96171.1 expression, respectively (Zhu et al., 2019). Furthermore, Yu et al. (2020) replicated the upregulated expression of this interactive network, and the positive correlation between functional connectivity of similar DMN regions and this lncRNA expression. These two pilot studies offered a clue for coexisting transcriptional and neuroimaging alterations related to AVH.

Up to present, seldom has focused on "trait" studies of SZ$\mathrm{AH}$ measuring the direct relation between cerebral expression of AH-related genes and neuroimaging features. Transcriptomeneuroimaging association studies are urgently required in the future to hopefully better the understanding of endophenotypes of AHs from both molecular and imaging angles.

\section{NEUROCOGNITIVE MODEL}

Various models of AHs have been put forward combining evidence of all levels. Generally, there are two main models of the hallucinatory pathogenesis concerning perceptual beliefs and inner speech.

According to the computational model of perception (Fletcher and Frith, 2009; Powers et al., 2017), perceptual beliefs, originating from prior experiences, influence one's sensation together with the actual sensory input, and hallucinations occur when the beliefs cause a percept without actual stimuli. Usually, perceptual beliefs are updated when prediction error 


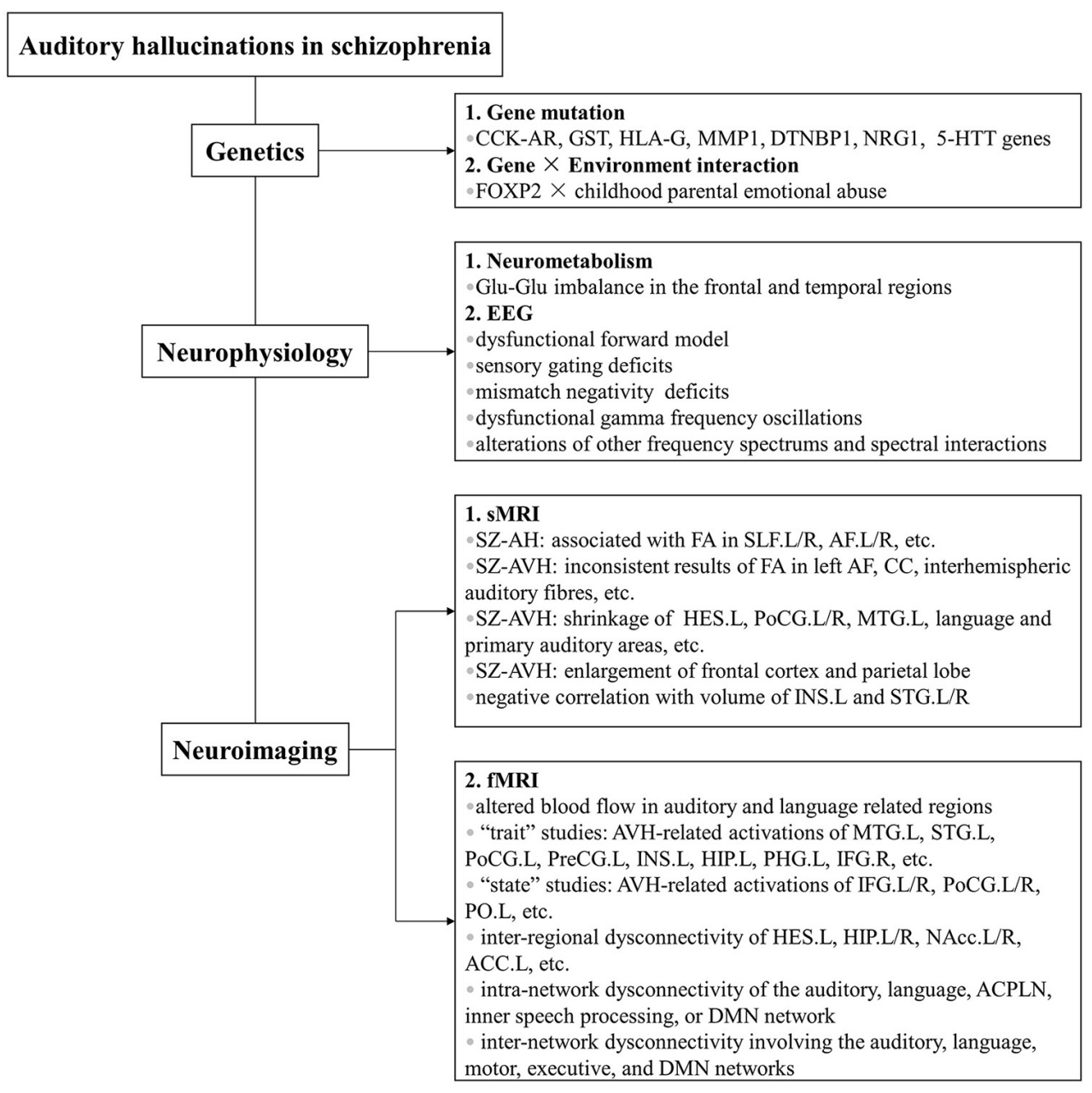

FIGURE 1 | Etiology of auditory hallucinations in schizophrenia from multidimensional levels. Previous work has utilized methods of various kinds, including genetic, neurophysiological, and neuroimaging studies. Main findings are summarized in the figure. Abbreviations: CCK-AR, cholecystokinin A receptor; GST, glutathione S-transferase; HLA-G, human leukocyte antigen-G; MMP1, matrix metalloproteinase 1; DTNBP1, dystrobrevin binding protein 1; NRG1, neuregulin 1; 5-HTT, serotonin transporter; FOXP2, forkhead box P2; EEG, electroencephalogram; sMRI, structural magnetic resonance imaging; SZ-AH, schizophrenia with auditory hallucinations; FA, fractional anisotropy; L, left; R, right; L/R, bilateral; SLF, bilateral superior longitudinal fasciculi; AF, arcuate fasciculi; SZ-AVH, schizophrenia with auditory verbal hallucinations; CC, corpus callosum; HES, Heschl's gyrus; PoCG, postcentral gyrus; MTG, middle temporal gyrus; INS, insula; STG, superior temporal gyrus; fMRI, functional magnetic resonance imaging; PreCG, precentral gyrus; HIP, hippocampus; PHG, parahippocampal gyrus; IFG, inferior frontal gyrus; PO, parietal operculum; NAcc, nucleus accumbens; ACC, anterior cingulate cortex; ACPLN, auditory cortex-posterior language network; DMN, default mode network.

occurs, which is the discrepancy between what is expected and what happens. Nevertheless, this mechanism has been demonstrated abnormal in schizophrenia (Corlett et al., 2007). In addition, hallucinating psychosis, regardless of the diagnosis, is less sensitive to the changes in contingency so as to have rigid perceptual beliefs and weighs more on perceptual beliefs than on actual sensory input, compared to non-hallucinating counterparts (Powers et al., 2017). Therefore, pathological perceptual beliefs may result in sensory perception even without objective stimuli, which further contributes to the formation of AHs among schizophrenia.

Another well-known model is inner speech model, which suggests that AHs arise on condition that inner speech (thinking in words) is perceived as someone else's, and this condition results from the deficits of self-monitoring (Frith and Done, 1988). Self-monitoring refers to one's ability to distinguish sensations evoked by one's own actions from those by external factors (Allen et al., 2007). This process is achieved, as what is mentioned earlier about the forward model, by the comparison between the corollary discharge and the actual auditory outcome to induce different neural responses to internally or externally generated motor action. Abnormal cerebral connectivity impairs the transmission of the corollary discharge in the forward model, resulting in the deficits of self-monitoring among schizophrenia (Stephan et al., 2009; Nazimek et al., 2012). Inner speech, in this case, could also be viewed as a motor action and is accompanied by a corollary discharge (Jack et al., 2019). Thus, failure to executive the function of self-monitoring leads 
to the misinterpretation of inner speech as if evoked by external source.

There have been other less popular models. The aberrant memory model postulates that failure to inhibit recalling and unintendedly activation of the memory system brings past traumatic memories to consciousness and generated unexpected intrusive thoughts. Early time trauma is closely connected to later life AVH. Consequently, memories that appear out of context contribute to the sensation of "otherness" and authorship from external side and then cause AVH (Tracy and Shergill, 2013). According to the spontaneous neural activity model (also called resting-state hypothesis), the occurrence of AVH is due to enhanced resting-state activity in the auditory cortex, aberrant modulation of the auditory cortex by anterior cortical midline regions (part of the DMN), and neural confusion between auditory cortical resting-state changes and stimulus-induced activity (Northoff and Qin, 2011). According to the expectationperception model (related to the self-monitoring mechanism), prefrontal regions are normally responsible for prediction and expectation of sensory input. As AHs happen, anatomical abnormalities in neurons, imbalance of neurotransmitter, and dysfunction of auditory cortex contribute to the deficient processing of prediction error, as a result of which the prediction from prefrontal regions becomes so unconstrained and vague that random fluctuations in spontaneous activity enhance the signal input and becomes a conscious percept in auditory cortex in the absence of external input (Nazimek et al., 2012).

\section{CONCLUSION}

\section{Findings From Multidimensional Levels}

With advances in our understanding of AHs, there lies an intricate fact that no single explanation so far has simply served for the full mechanism underlying these symptoms. Fruitful findings in the past decades have shown that the pathogenesis of AHs in schizophrenia has independent genetic basis and neural substrates revealed by multidimensional levels. Based on what is summarized in this review, AHs are influenced by multiple gene and gene $x$ environment interactions from genetic level, by glutamate level imbalance from neurometabolic level, by dysfunctional forward model, sensory gating deficits, MMN deficits, dysfunctional $\gamma$ frequency oscillations, and alterations of other frequency spectrums and spectral interactions from EEG level, by fasciculus alterations of white matter and morphological changes from sMRI level, and by altered cerebral blood flow, abnormal cerebral activations, and dysfunctional brain connectivity of interregion, intranetwork, and internetwork from fMRI level (summarized in Figure 1).

However, there are several aspects worthy of discussion in order to improve current research. First, small sample size, heterogeneous patients' condition, and diversified methodologies and study designs make current evidences less easy to be replicated. One priority of future studies is to enlarge sample size and devise comparable design to allow confirmation of existing findings. Second, other types of AHs apart from the verbal type warrant more attention in the future as they are seldom studied up to present. And multimodal studies are also welcomed to combine evidences from different aspects. Third, a body of previous studies was conducted in schizophrenia with auditory (verbal) hallucinations and healthy individuals, which makes it difficult to tell whether the group differences result from the hallucinatory experience or the disease itself. As a result, "trait" study that directly compares SZ-AH and SZ-non-AH or compares SZ-AVH and SZ-non-AVH is more preferred to rule out the possible influence of the clinical state. Fourthly, "state" study that compares hallucinatory with nonhallucinatory period using self-control is tremendously scarce, probably because the ongoing hallucinatory state is relatively tricky to fully capture, and fMRI and EEG are ones of the few tools capable of distinguishing "trait" and "state" studies. Therefore, study of this kind is also wanted in the future. Last but not least, current scanty of transcriptional-neuroimaging studies of AHs calls for more endeavor in this field.

\section{Hypothesis 1. Schizophrenia: Auditory Hallucinations vs. Auditory Verbal Hallucinations}

Although plenty of studies focus on AHs in general, there is still an abundant load of work specifically on the verbal type, $\mathrm{AVH}$, probably due to the verbal type as a core positive symptom of schizophrenia. And previous research of the general and the verbal type has yielded inconsistent results.

In genetic research, gene candidates and transcriptomic studies are solely found in SZ-AH, whereas gene $\times$ environment interaction is solely found in SZ-AVH. In neurometabolic research, interregional Glu-Glu imbalance was concluded from combined evidence of both general AHs and the verbal type, and other neurometabolic studies have been mainly conducted in SZ-AH. In EEG research, sensory gating deficits are solely found in SZ-AVH, whereas other results are mainly found in SZAH. In sMRI research, although cerebral alterations are found from combined evidence of both SZ-AH and SZ-AVH, two groups have yielded inconsistent results. For example, regarding DTI study, "trait" studies showed SZ-AH lower FA in bilateral superior longitudinal fasciculi and arcuate fasciculi. Differently in SZ-AVH, although FA changes in other fasciculi were reported, those in superior longitudinal fasciculi were not, and results concerning arcuate fasciculi were inconclusive. For another example, regarding morphological study, scanty "trait" study in SZ-AH showed larger volumes of temporal white matter, frontal gray matter, and temporal gray matter, which did not accord with findings in SZ-AVH. In fMRI research, SZ-AVH had specific regional activation replicated by meta-analyses. While SZ-AH had enhanced functional connectivity of regions related to mesolimbic pathway, SZ-AVH of regions related to language and auditory system. While SZ-AH had dysconnectivity within the auditory network, SZ-AVH had dysconnectivity not only within the language, the inner speech processing, and the DMNs, but also between cerebral networks.

Therefore, our first hypothesis is that AVH may have independent mechanism from other types of AHs. In fact, one reason for poor replicability of current studies could be 
that AHs of different subtypes are treated as a whole group during research. Currently, comparative studies among subtypes of AHs in schizophrenia, although scarce, have shown that different subtypes may have distinct phenomenological features and functional brain patterns. McCarthy-Jones et al. (2014b) classified AHs into constant commanding and commenting $\mathrm{AVH}$, replay $\mathrm{AVH}$, own though AVH, and non-verbal AHs. One study found schizophrenia with non-verbal AHs showed higher global functional connectivity density in bilateral superior temporal gyri and lower global functional connectivity density (gFCD) in bilateral prefrontal cortex, inferior frontal lobe, and occipital lobe (Zhuo et al., 2020). These preliminary evidences have supported our hypothesis. We suggest future research should separate AHs into different groups to make subtype comparisons.

\section{Hypothesis 2. Auditory Hallucinations: From Genotype to Phenotype}

Our second hypothesis is that AHs are heterogeneous symptoms rooted deeply in genetic background. Mediating genes cause neurophysiological alterations and structural and functional cerebral changes, which further cause distinct clinical features of AHs in schizophrenia. Based on existing genetic research, $D T N B P 1$ is a promising gene candidate for AHs. Its function is related to regulation of glutamate level, which is consistent with neurometabolic findings of interregional Glu-Glu imbalance (especially in the frontal and temporal regions). Its mRNA expression is lower in superior temporal gyrus and hippocampus. Regarding superior temporal gyrus, correlation between glutamate level and AHs based on neurometabolic study was found in this area. Morphological volume changes based on sMRI study and aberrant activation and functional connectivity changes based on fMRI study were also found in this area. Regarding hippocampus, structural and functional changes were also found based on sMRI and fMRI study. We hypothesized that reduced cerebral DTNBP1 expression leads to glutamate level changes and structural and functional changes of superior

\section{REFERENCES}

Akarsu, S., Torun, D., Bolu, A., Erdem, M., Kozan, S., Ak, M., et al. (2014). Mitochondrial complex I and III gene mRNA levels in schizophrenia, and their relationship with clinical features. J. Mol. Psychiatry 2:6. doi: 10.1186/s40303014-0006-9

Allen, P., Aleman, A., and McGuire, P. K. (2007). Inner speech models of auditory verbal hallucinations: evidence from behavioural and neuroimaging studies. Int. Rev. Psychiatry 19, 407-415. doi: 10.1080/09540260701486498

Alonso-Solis, A., Vives-Gilabert, Y., Grasa, E., Portella, M. J., Rabella, M., Sauras, R. B., et al. (2015). Resting-state functional connectivity alterations in the default network of schizophrenia patients with persistent auditory verbal hallucinations. Schizophr. Res. 161, 261-268. doi: 10.1016/j.schres.2014. 10.047

Amad, A., Cachia, A., Gorwood, P., Pins, D., Delmaire, C., Rolland, B., et al. (2014). The multimodal connectivity of the hippocampal complex in auditory and visual hallucinations. Mol. Psychiatry 19, 184-191. doi: 10.1038/mp. 2012.181

American Psychiatric Association (2013). Diagnostic and Statistical Manual of Mental Disorders, Fifth Edn. Arlington, TX: American Psychiatric Association.

Angelopoulos, E., Koutsoukos, E., Maillis, A., Papadimitriou, G. N., and Stefanis, C. (2011). Cortical interactions during the experience of auditory verbal temporal gyrus and hippocampus. Our hypothesis is according to current multidimensional research, but these evidences are not collected from the same patients. Research to date often utilizes a single scale, resulting in incoherent understanding of the etiology. We suggest future research use a multimode framework that uses multiscale data, from genomics, transcriptomic, neurophysiology, to neuroimaging (i.e., from genotype to phenotype). Therefore, multidimensional research conducted on one batch of patients is strongly preferred to confirm the effect of genetic variants on brain structures that contribute to AHs.

Investigations on pathogenesis of mental disorders and related phenomenology have always been a challenge hard to conquer, and AHs in schizophrenia are no exception. Although great advancement has been accomplished in the past decades, much more endeavor still needs to be made in the future. Hopefully, discovery of mechanisms underlying AHs will contribute to a deeper understanding of the essence of this symptom and will have substantial implications for clinical practice.

\section{AUTHOR CONTRIBUTIONS}

XS and JT conceived the study. XS completed the first draft. All authors contributed to the literature research and analyses and approved the final manuscript.

\section{FUNDING}

This research was supported by the National Natural Science Foundation of China (grant numbers 81871057 and 8217050237, JT) and in part by the Japan Science and Technology Agency under grant ACT-X (grant number JPMJAX190D and Moonshot R\&D grant number JPMJMS2011, LG). The funder had no role in study design, data collection and analysis, decision to publish, or preparation of the manuscript.

hallucinations. J. Neuropsychiatry Clin. Neurosci. 23, 287-293. doi: 10.1176/ appi.neuropsych.23.3.287

Barta, P. E., Pearlson, G. D., Powers, R. E., Richards, S. S., and Tune, L. E. (1990). Auditory hallucinations and smaller superior temporal gyral volume in schizophrenia. Am. J. Psychiatry 147, 1457-1462. doi: 10.1176/ajp.147.11.1457

Bauer, S. M., Schanda, H., Karakula, H., Olajossy-Hilkesberger, L., Rudaleviciene, P., Okribelashvili, N., et al. (2011). Culture and the prevalence of hallucinations in schizophrenia. Compr. Psychiatry 52, 319-325.

Beneyto, M., Abbott, A., Hashimoto, T., and Lewis, D. A. (2011). Lamina-specific alterations in cortical GABA(A) receptor subunit expression in schizophrenia. Cereb. Cortex 21, 999-1011. doi: 10.1093/cercor/bhq169

Busatto, G. F., David, A. S., Costa, D. C., Ell, P. J., Pilowsky, L. S., Lucey, J. V., et al. (1995). Schizophrenic auditory hallucinations are associated with increased regional cerebral blood flow during verbal memory activation in a study using single photon emission computed tomography. Psychiatry Res. 61, 255-264. doi: 10.1016/0925-4927(95)02750-r

Carlsson, A., Waters, N., Holm-Waters, S., Tedroff, J., Nilsson, M., and Carlsson, M. L. (2001). Interactions between monoamines, glutamate, and GABA in schizophrenia: new evidence. Annu. Rev. Pharmacol. Toxicol. 41, 237-260. doi: 10.1146/annurev.pharmtox.41.1.237

Catani, M., Craig, M. C., Forkel, S. J., Kanaan, R., Picchioni, M., Toulopoulou, T., et al. (2011). Altered integrity of perisylvian language pathways in 
schizophrenia: relationship to auditory hallucinations. Biol. Psychiatry 70, 1143-1150. doi: 10.1016/j.biopsych.2011.06.013

Chang, X., Collin, G., Xi, Y., Cui, L., Scholtens, L. H., Sommer, I. E., et al. (2017). Resting-state functional connectivity in medication-naive schizophrenia patients with and without auditory verbal hallucinations: a preliminary report. Schizophr. Res. 188, 75-81. doi: 10.1016/j.schres.2017.01. 024

Chawla, N., Deep, R., Khandelwal, S. K., and Garg, A. (2019). Reduced integrity of superior longitudinal fasciculus and arcuate fasciculus as a marker for auditory hallucinations in schizophrenia: a DTI tractography study. Asian J. Psychiatr. 44, 179-186. doi: 10.1016/j.ajp.2019.07.043

Cheah, S. Y., Lawford, B. R., Young, R. M., Morris, C. P., and Voisey, J. (2015). Dysbindin (DTNBP1) variants are associated with hallucinations in schizophrenia. Eur. Psychiatry 30, 486-491. doi: 10.1016/j.eurpsy.2015.01.008

Chen, X., Liang, S., Pu, W., Song, Y., Mwansisya, T. E., Yang, Q., et al. (2015). Reduced cortical thickness in right Heschl's gyrus associated with auditory verbal hallucinations severity in first-episode schizophrenia. BMC Psychiatry 15:152. doi: 10.1186/s12888-015-0546-2

Chiang, Y. H., Beckstead, J. W., Lo, S. C., and Yang, C. Y. (2018). Association of auditory hallucination and anxiety symptoms with depressive symptoms in patients with schizophrenia: a three-month follow-up. Arch. Psychiatr. Nurs. 32, $585-590$.

Cierpka, M., Wolf, N. D., Kubera, K. M., Schmitgen, M. M., Vasic, N., Frasch, K., et al. (2017). Cerebellar contributions to persistent auditory verbal hallucinations in patients with schizophrenia. Cerebellum 16, 964-972. doi: 10.1007/s12311-017-0874-5

Corlett, P. R., Murray, G. K., Honey, G. D., Aitken, M. R., Shanks, D. R., Robbins, T. W., et al. (2007). Disrupted prediction-error signal in psychosis: evidence for an associative account of delusions. Brain 130(Pt. 9), 2387-2400. doi: 10.1093/ brain/awm 173

Cui, L. B., Chen, G., Xu, Z. L., Liu, L., Wang, H. N., Guo, L., et al. (2017a). Cerebral blood flow and its connectivity features of auditory verbal hallucinations in schizophrenia: a perfusion study. Psychiatry Res. Neuroimaging 260, 53-61. doi: 10.1016/j.pscychresns.2016.12.006

Cui, L. B., Liu, L., Guo, F., Chen, Y. C., Chen, G., Xi, M., et al. (2017b). Disturbed brain activity in resting-state networks of patients with first-episode schizophrenia with auditory verbal hallucinations: a cross-sectional functional MR imaging study. Radiology 283, 810-819. doi: 10.1148/radiol.2016160938

Cui, Y., Liu, B., Song, M., Lipnicki, D. M., Li, J., Xie, S., et al. (2018). Auditory verbal hallucinations are related to cortical thinning in the left middle temporal Gyrus of patients with schizophrenia. Psychol. Med. 48, 115-122. doi: 10.1017/ s0033291717001520

Curcic-Blake, B., Bais, L., Sibeijn-Kuiper, A., Pijnenborg, H. M., Knegtering, H., Liemburg, E., et al. (2017). Glutamate in dorsolateral prefrontal cortex and auditory verbal hallucinations in patients with schizophrenia: a (1)H MRS study. Prog. Neuropsychopharmacol. Biol. Psychiatry 78, 132-139. doi: 10.1016/ j.pnpbp.2017.05.020

Curcic-Blake, B., Nanetti, L., van der Meer, L., Cerliani, L., Renken, R., Pijnenborg, G. H., et al. (2015). Not on speaking terms: hallucinations and structural network disconnectivity in schizophrenia. Brain Struct. Funct. 220, 407-418. doi: 10.1007/s00429-013-0663-y

Duncan, C. E., Webster, M. J., Rothmond, D. A., Bahn, S., Elashoff, M., and Shannon Weickert, C. (2010). Prefrontal GABA(A) receptor alpha-subunit expression in normal postnatal human development and schizophrenia. J. Psychiatr Res. 44, 673-681. doi: 10.1016/j.jpsychires.2009.12.007

Eliades, S. J., and Wang, X. (2003). Sensory-motor interaction in the primate auditory cortex during self-initiated vocalizations. J. Neurophysiol. 89, 21942207. doi: $10.1152 /$ jn. 00627.2002

Enwright, J. F., and Lewis, D. A. (2021). Similarities in cortical transcriptome alterations between schizophrenia and bipolar disorder are related to the presence of psychosis. Schizophr. Bull. 47, 1442-1451. doi: 10.1093/schbul/ sbaa 195

Faugere, M., Micoulaud-Franchi, J. A., Boyer, L., Cermolacce, M., Richieri, R., Faget, C., et al. (2016). Does sensory gating have a protective effect against hallucinatory behavior in schizophrenia? Clin. Neurophysiol. 127, 1746-1748. doi: 10.1016/j.clinph.2015.10.031

Fisher, D. J., Grant, B., Smith, D. M., Borracci, G., Labelle, A., and Knott, V. J. (2011). Effects of auditory hallucinations on the mismatch negativity (MMN) in schizophrenia as measured by a modified 'optimal' multi-feature paradigm. Int. J. Psychophysiol. 81, 245-251. doi: 10.1016/j.ijpsycho.2011. 06.018

Fisher, D. J., Labelle, A., and Knott, V. J. (2008). The right profile: mismatch negativity in schizophrenia with and without auditory hallucinations as measured by a multi-feature paradigm. Clin. Neurophysiol. 119, 909-921. doi: 10.1016/j.clinph.2007.12.005

Fisher, D. J., Labelle, A., and Knott, V. J. (2012). Alterations of mismatch negativity (MMN) in schizophrenia patients with auditory hallucinations experiencing acute exacerbation of illness. Schizophr. Res. 139, 237-245. doi: 10.1016/j.schres. 2012.06.004

Fisher, D. J., Smith, D. M., Labelle, A., and Knott, V. J. (2014). Attenuation of mismatch negativity (MMN) and novelty P300 in schizophrenia patients with auditory hallucinations experiencing acute exacerbation of illness. Biol. Psychol. 100, 43-49. doi: 10.1016/j.biopsycho.2014.05.005

Fletcher, P. C., and Frith, C. D. (2009). Perceiving is believing: a Bayesian approach to explaining the positive symptoms of schizophrenia. Nat. Rev. Neurosci. 10, 48-58. doi: 10.1038/nrn2536

Ford, J. M., Roach, B. J., Faustman, W. O., and Mathalon, D. H. (2007). Synch before you speak: auditory hallucinations in schizophrenia. Am. J. Psychiatry 164, 458-466. doi: 10.1176/ajp.2007.164.3.458

Fox, M. D., and Raichle, M. E. (2007). Spontaneous fluctuations in brain activity observed with functional magnetic resonance imaging. Nat. Rev. Neurosci. 8, 700-711. doi: 10.1038/nrn2201

Freedman, R., Adler, L. E., Gerhardt, G. A., Waldo, M., Baker, N., Rose, G. M., et al. (1987). Neurobiological studies of sensory gating in schizophrenia. Schizophr. Bull. 13, 669-678. doi: 10.1093/schbul/13.4.669

Frith, C. D., and Done, D. J. (1988). Towards a neuropsychology of schizophrenia. Br. J. Psychiatry 153, 437-443. doi: 10.1192/bjp.153.4.437

Garcia-Marti, G., Aguilar, E. J., Lull, J. J., Marti-Bonmati, L., Escarti, M. J., Manjon, J. V., et al. (2008). Schizophrenia with auditory hallucinations: a voxel-based morphometry study. Prog. Neuropsychopharmacol. Biol. Psychiatry 32, 72-80. doi: 10.1016/j.pnpbp.2007.07.014

Gaser, C., Nenadic, I., Volz, H. P., Buchel, C., and Sauer, H. (2004). Neuroanatomy of "hearing voices": a frontotemporal brain structural abnormality associated with auditory hallucinations in schizophrenia. Cereb. Cortex 14, 91-96. doi: $10.1093 /$ cercor/bhg107

Gavrilescu, M., Rossell, S., Stuart, G. W., Shea, T. L., Innes-Brown, H., Henshall, K., et al. (2010). Reduced connectivity of the auditory cortex in patients with auditory hallucinations: a resting state functional magnetic resonance imaging study. Psychol. Med. 40, 1149-1158. doi: 10.1017/s0033291709991632

Gibbons, A. S., Bell, L. M., Udawela, M., and Dean, B. (2020). mRNA expression of the P5 ATPase ATP13A4 is increased in Broca's area from subjects with schizophrenia. World J. Biol. Psychiatry 21, 402-408. doi: 10.1080/15622975. 2018.1548781

Gilabert-Juan, J., Sáez, A. R., Lopez-Campos, G., Sebastiá-Ortega, N., GonzálezMartínez, R., Costa, J., et al. (2015). Semaphorin and plexin gene expression is altered in the prefrontal cortex of schizophrenia patients with and without auditory hallucinations. Psychiatry Res. 229, 850-857. doi: 10.1016/j.psychres. 2015.07.074

Griskova-Bulanova, I., Hubl, D., van Swam, C., Dierks, T., and Koenig, T. (2016). Early- and late-latency gamma auditory steady-state response in schizophrenia during closed eyes: does hallucination status matter? Clin. Neurophysiol. 127, 2214-2221. doi: 10.1016/j.clinph.2016.02.009

Guo, Q., Hu, Y., Zeng, B., Tang, Y., Li, G., Zhang, T., et al. (2020). Parietal memory network and default mode network in first-episode drug-naive schizophrenia: associations with auditory hallucination. Hum. Brain Mapp. 41, 1973-1984. doi: $10.1002 / \mathrm{hbm} .24923$

Heckers, S. (2001). Neuroimaging studies of the hippocampus in schizophrenia. Hippocampus 11, 520-528. doi: 10.1002/hipo.1068

Heils, A., Teufel, A., Petri, S., Stober, G., Riederer, P., Bengel, D., et al. (1996). Allelic variation of human serotonin transporter gene expression. J. Neurochem. 66, 2621-2624. doi: 10.1046/j.1471-4159.1996.66062621.x

Heinks-Maldonado, T. H., Mathalon, D. H., Houde, J. F., Gray, M., Faustman, W. O., and Ford, J. M. (2007). Relationship of imprecise corollary discharge in schizophrenia to auditory hallucinations. Arch. Gen. Psychiatry 64, 286-296. doi: 10.1001/archpsyc.64.3.286

Hirano, Y., Hirano, S., Maekawa, T., Obayashi, C., Oribe, N., Monji, A., et al. (2010). Auditory gating deficit to human voices in schizophrenia: a MEG study. Schizophr. Res. 117, 61-67. doi: 10.1016/j.schres.2009.09.003 
Hirano, Y., Oribe, N., Kanba, S., Onitsuka, T., Nestor, P. G., and Spencer, K. M. (2015). Spontaneous gamma activity in schizophrenia. JAMA Psychiatry 72, 813-821. doi: 10.1001/jamapsychiatry.2014.2642

Hjelmervik, H., Craven, A. R., Sinceviciute, I., Johnsen, E., Kompus, K., Bless, J. J., et al. (2019). Intra-Regional Glu-GABA vs inter-regional glu-glu imbalance: a 1 H-MRS study of the neurochemistry of auditory verbal hallucinations in schizophrenia. Schizophr. Bull. 46, 633-642. doi: 10.1093/schbul/sbz099

Hoffman, R. E., Fernandez, T., Pittman, B., and Hampson, M. (2011). Elevated functional connectivity along a corticostriatal loop and the mechanism of auditory/verbal hallucinations in patients with schizophrenia. Biol. Psychiatry 69, 407-414. doi: 10.1016/j.biopsych.2010.09.050

Huang, J., Liu, F., Wang, B., Tang, H., Teng, Z., Li, L., et al. (2019). Central and peripheral changes in FOS expression in schizophrenia based on genome-wide gene expression. Front. Genet. 10:232. doi: 10.3389/fgene.2019.00232

Hubl, D., Dougoud-Chauvin, V., Zeller, M., Federspiel, A., Boesch, C., Strik, W., et al. (2010). Structural analysis of Heschl's gyrus in schizophrenia patients with auditory hallucinations. Neuropsychobiology 61, 1-9. doi: 10.1159/000258637

Hubl, D., Koenig, T., Strik, W., Federspiel, A., Kreis, R., Boesch, C., et al. (2004). Pathways that make voices: white matter changes in auditory hallucinations. Arch. Gen. Psychiatry 61, 658-668. doi: 10.1001/archpsyc.61.7.658

Hugdahl, K., and Sommer, I. E. (2018). Auditory verbal hallucinations in schizophrenia from a levels of explanation perspective. Schizophr. Bull. 44, 234-241. doi: 10.1093/schbul/sbx142

Hugdahl, K., Craven, A. R., Nygard, M., Loberg, E. M., Berle, J. O., Johnsen, E., et al. (2015). Glutamate as a mediating transmitter for auditory hallucinations in schizophrenia: a (1)H MRS study. Schizophr. Res. 161, 252-260. doi: 10.1016/ j.schres.2014.11.015

Jack, B. N., Le Pelley, M. E., Han, N., Harris, A. W. F., Spencer, K. M., and Whitford, T. J. (2019). Inner speech is accompanied by a temporally-precise and content-specific corollary discharge. Neuroimage 198, 170-180. doi: 10.1016/j. neuroimage.2019.04.038

Jardri, R., Pouchet, A., Pins, D., and Thomas, P. (2011). Cortical activations during auditory verbal hallucinations in schizophrenia: a coordinate-based metaanalysis. Am. J. Psychiatry 168, 73-81. doi: 10.1176/appi.ajp.2010.09101522

Javitt, D. C., and Freedman, R. (2015). Sensory processing dysfunction in the personal experience and neuronal machinery of schizophrenia. Am. J. Psychiatry 172, 17-31. doi: 10.1176/appi.ajp.2014.13121691

Kim, J. W., Kang, W. S., Lee, S. M., Kim, S. K., Park, H. J., Gwak, G. H., et al. (2012). Association between a synonymous SNP (rs470558, Ala216Ala) of MMP1 and schizophrenia with auditory hallucinations in Korean population. Mol. Cell. Toxicol. 8, 297-302. doi: 10.1007/s13273-012-0036-2

Kim, J. W., Lee, Y. S., Cho, E. Y., Jang, Y. L., Park, D. Y., Choi, K. S., et al. (2006). Linkage and association of schizophrenia with genetic variations in the locus of neuregulin 1 in Korean population. Am. J. Med. Genet. B Neuropsychiatr. Genet. 141b, 281-286. doi: 10.1002/ajmg.b.30209

Knochel, C., Oertel-Knochel, V., Schonmeyer, R., Rotarska-Jagiela, A., van de Ven, V., Prvulovic, D., et al. (2012). Interhemispheric hypoconnectivity in schizophrenia: fiber integrity and volume differences of the corpus callosum in patients and unaffected relatives. Neuroimage 59, 926-934. doi: 10.1016/j. neuroimage.2011.07.088

Koenig, T., van Swam, C., Dierks, T., and Hubl, D. (2012). Is gamma band EEG synchronization reduced during auditory driving in schizophrenia patients with auditory verbal hallucinations? Schizophr. Res. 141, 266-270. doi: 10.1016/j. schres.2012.07.016

Kompus, K., Westerhausen, R., and Hugdahl, K. (2011). The "paradoxical" engagement of the primary auditory cortex in patients with auditory verbal hallucinations: a meta-analysis of functional neuroimaging studies. Neuropsychologia 49, 3361-3369. doi: 10.1016/j.neuropsychologia.2011.08.010

Koutsoukos, E., Angelopoulos, E., Maillis, A., Papadimitriou, G. N., and Stefanis, C. (2013). Indication of increased phase coupling between theta and gamma EEG rhythms associated with the experience of auditory verbal hallucinations. Neurosci. Lett. 534, 242-245. doi: 10.1016/j.neulet.2012.12.005

Koyanagi, A., Stickley, A., and Haro, J. M. (2015). Subclinical psychosis and suicidal behavior in England: findings from the 2007 Adult Psychiatric Morbidity Survey. Schizophr. Res. 168, 62-67. doi: 10.1016/j.schres.2015.07.041

Kubera, K. M., Sambataro, F., Vasic, N., Wolf, N. D., Frasch, K., Hirjak, D., et al. (2014). Source-based morphometry of gray matter volume in patients with schizophrenia who have persistent auditory verbal hallucinations. Prog.
Neuropsychopharmacol. Biol. Psychiatry 50, 102-109. doi: 10.1016/j.pnpbp. 2013.11.015

Kuga, H., Onitsuka, T., Hirano, Y., Nakamura, I., Oribe, N., Mizuhara, H., et al. (2016). Increased BOLD signals elicited by high gamma auditory stimulation of the left auditory cortex in acute state schizophrenia. EBioMedicine 12, 143-149. doi: 10.1016/j.ebiom.2016.09.008

Kühn, S., and Gallinat, J. (2012). Quantitative meta-analysis on state and trait aspects of auditory verbal hallucinations in schizophrenia. Schizophr. Bull. 38, 779-786. doi: $10.1093 /$ schbul/sbq152

Kurian, S. M., Le-Niculescu, H., Patel, S. D., Bertram, D., Davis, J., Dike, C., et al. (2011). Identification of blood biomarkers for psychosis using convergent functional genomics. Mol. Psychiatry 16, 37-58. doi: 10.1038/mp.2009.117

Laroi, F., Sommer, I. E., Blom, J. D., Fernyhough, C., Ffytche, D. H., Hugdahl, K., et al. (2012). The characteristic features of auditory verbal hallucinations in clinical and nonclinical groups: state-of-the-art overview and future directions. Schizophr. Bull. 38, 724-733.

Lavigne, K. M., and Woodward, T. S. (2018). Hallucination- and speechspecific hypercoupling in frontotemporal auditory and language networks in schizophrenia using combined task-based fMRI data: an fBIRN study. Hum. Brain Mapp. 39, 1582-1595. doi: 10.1002/hbm.23934

Lavigne, K. M., Rapin, L. A., Metzak, P. D., Whitman, J. C., Jung, K., Dohen, M., et al. (2015). Left-dominant temporal-frontal hypercoupling in schizophrenia patients with hallucinations during speech perception. Schizophr. Bull. 41, 259-267. doi: 10.1093/schbul/sbu004

Lee, K., Yoshida, T., Kubicki, M., Bouix, S., Westin, C. F., Kindlmann, G., et al. (2009). Increased diffusivity in superior temporal gyrus in patients with schizophrenia: a Diffusion Tensor Imaging study. Schizophr. Res. 108, 33-40. doi: 10.1016/j.schres.2008.11.024

Lee, S. H., Choo, J. S., Im, W. Y., and Chae, J. H. (2008). Nonlinear analysis of electroencephalogram in schizophrenia patients with persistent auditory hallucination. Psychiatry Investig. 5, 115-120. doi: 10.4306/pi.2008.5.2.115

Lee, S. H., Wynn, J. K., Green, M. F., Kim, H., Lee, K. J., Nam, M., et al. (2006). Quantitative EEG and low resolution electromagnetic tomography (LORETA) imaging of patients with persistent auditory hallucinations. Schizophr. Res. 83, 111-119. doi: 10.1016/j.schres.2005.11.025

Lefebvre, S., Demeulemeester, M., Leroy, A., Delmaire, C., Lopes, R., Pins, D., et al. (2016). Network dynamics during the different stages of hallucinations in schizophrenia. Hum. Brain Mapp. 37, 2571-2586. doi: 10.1002/hbm.23197

Leroux, E., Delcroix, N., and Dollfus, S. (2017). Abnormalities of language pathways in schizophrenia patients with and without a lifetime history of auditory verbal hallucinations: a DTI-based tractography study. World J. Biol. Psychiatry 18, 528-538. doi: 10.1080/15622975.2016.1274053

Levitan, C., Ward, P. B., and Catts, S. V. (1999). Superior temporal gyral volumes and laterality correlates of auditory hallucinations in schizophrenia. Biol. Psychiatry 46, 955-962. doi: 10.1016/s0006-3223(98)00373-4

Li, B., Cui, L. B., Xi, Y. B., Friston, K. J., Guo, F., Wang, H. N., et al. (2017). Abnormal effective connectivity in the brain is involved in auditory verbal hallucinations in schizophrenia. Neurosci. Bull. 33, 281-291. doi: 10.1007/ s12264-017-0101-x

Liegeois, F., Baldeweg, T., Connelly, A., Gadian, D. G., Mishkin, M., and VarghaKhadem, F. (2003). Language fMRI abnormalities associated with FOXP2 gene mutation. Nat. Neurosci. 6, 1230-1237. doi: 10.1038/nn1138

Lim, A., Hoek, H. W., Deen, M. L., and Blom, J. D. (2016). Prevalence and classification of hallucinations in multiple sensory modalities in schizophrenia spectrum disorders. Schizophr. Res. 176, 493-499.

Liu, L., Cui, L. B., Xi, Y. B., Wang, X. R., Liu, Y. C., Xu, Z. L., et al. (2019). Association between connectivity of hippocampal sub-regions and auditory verbal hallucinations in schizophrenia. Front. Neurosci. 13:424. doi: 10.3389/ fnins.2019.00424

Maijer, K., Begemann, M. J. H., Palmen, S., Leucht, S., and Sommer, I. E. C. (2018). Auditory hallucinations across the lifespan: a systematic review and meta-analysis. Psychol. Med. 48, 879-888. doi: 10.1017/s0033291717002367

Malhotra, A. K., Goldman, D., Mazzanti, C., Clifton, A., Breier, A., and Pickar, D. (1998). A functional serotonin transporter (5-HTT) polymorphism is associated with psychosis in neuroleptic-free schizophrenics. Mol. Psychiatry 3 , 328-332. doi: 10.1038/sj.mp.4000412

Marques, T. R., Ashok, A. H., Angelescu, I., Borgan, F., Myers, J., LingfordHughes, A., et al. (2020). GABA-A receptor differences in schizophrenia: a 
positron emission tomography study using [(11)C]Ro154513. Mol. Psychiatry 26, 2616-2625. doi: 10.1038/s41380-020-0711-y

Martinez-Granados, B., Brotons, O., Martinez-Bisbal, M. C., Celda, B., MartiBonmati, L., Aguilar, E. J., et al. (2008). Spectroscopic metabolomic abnormalities in the thalamus related to auditory hallucinations in patients with schizophrenia. Schizophr. Res. 104, 13-22. doi: 10.1016/j.schres.2008.05.025

McCarthy-Jones, S., Green, M. J., Scott, R. J., Tooney, P. A., Cairns, M. J., Wu, J. Q., et al. (2014a). Preliminary evidence of an interaction between the FOXP2 gene and childhood emotional abuse predicting likelihood of auditory verbal hallucinations in schizophrenia. J. Psychiatr Res. 50, 66-72. doi: 10.1016/j. jpsychires.2013.11.012

McCarthy-Jones, S., Oestreich, L. K., and Whitford, T. J. (2015). Reduced integrity of the left arcuate fasciculus is specifically associated with auditory verbal hallucinations in schizophrenia. Schizophr. Res. 162, 1-6. doi: 10.1016/j.schres. 2014.12.041

McCarthy-Jones, S., Trauer, T., Mackinnon, A., Sims, E., Thomas, N., and Copolov, D. (2014b). A new phenomenological survey of auditory hallucinations: evidence for subtypes and implications for theory and practice. Schizophr. Bull. 40, 231-235. doi: 10.1093/schbul/sbs156

McGuire, P. K., Shah, G. M., and Murray, R. M. (1993). Increased blood flow in Broca's area during auditory hallucinations in schizophrenia. Lancet 342, 703-706. doi: 10.1016/0140-6736(93)91707-s

McGuire, P. K., Silbersweig, D. A., Wright, I., Murray, R. M., David, A. S., Frackowiak, R. S., et al. (1995). Abnormal monitoring of inner speech: a physiological basis for auditory hallucinations. Lancet 346, 596-600. doi: 10. 1016/s0140-6736(95)91435-8

Melbourne, J. K., Rosen, C., Feiner, B., and Sharma, R. P. (2018). C4A mRNA expression in PBMCs predicts the presence and severity of delusions in schizophrenia and bipolar disorder with psychosis. Schizophr. Res. 197, 321327. doi: 10.1016/j.schres.2018.01.018

Modinos, G., Costafreda, S. G., van Tol, M. J., McGuire, P. K., Aleman, A., and Allen, P. (2013). Neuroanatomy of auditory verbal hallucinations in schizophrenia: a quantitative meta-analysis of voxel-based morphometry studies. Cortex 49, 1046-1055. doi: 10.1016/j.cortex.2012.01.009

Modinos, G., Vercammen, A., Mechelli, A., Knegtering, H., McGuire, P. K., and Aleman, A. (2009). Structural covariance in the hallucinating brain: a voxel-based morphometry study. J. Psychiatry Neurosci. 34, $465-469$.

Morch-Johnsen, L., Nesvag, R., Jorgensen, K. N., Lange, E. H., Hartberg, C. B., Haukvik, U. K., et al. (2017). Auditory cortex characteristics in schizophrenia: associations with auditory hallucinations. Schizophr. Bull. 43, 75-83. doi: 10. 1093/schbul/sbw130

Mou, X., Bai, F., Xie, C., Shi, J., Yao, Z., Hao, G., et al. (2013). Voice recognition and altered connectivity in schizophrenic patients with auditory hallucinations. Prog. Neuropsychopharmacol. Biol. Psychiatry 44, 265-270. doi: 10.1016/j. pnpbp.2013.03.006

Mulert, C., Kirsch, V., Pascual-Marqui, R., McCarley, R. W., and Spencer, K. M. (2011). Long-range synchrony of gamma oscillations and auditory hallucination symptoms in schizophrenia. Int. J. Psychophysiol. 79, 55-63. doi: 10.1016/j.ijpsycho.2010.08.004

Mulert, C., Kirsch, V., Whitford, T. J., Alvarado, J., Pelavin, P., McCarley, R. W., et al. (2012). Hearing voices: a role of interhemispheric auditory connectivity? World J. Biol. Psychiatry 13, 153-158. doi: 10.3109/15622975.2011.570789

Naatanen, R., Paavilainen, P., Rinne, T., and Alho, K. (2007). The mismatch negativity (MMN) in basic research of central auditory processing: a review. Clin. Neurophysiol. 118, 2544-2590. doi: 10.1016/j.clinph.2007.04.026

Nazimek, J. M., Hunter, M. D., and Woodruff, P. W. (2012). Auditory hallucinations: expectation-perception model. Med. Hypotheses 78, 802-810. doi: 10.1016/j.mehy.2012.03.014

Nenadic, I., Dietzek, M., Langbein, K., Rzanny, R., Gussew, A., Reichenbach, J. R., et al. (2014). Superior temporal metabolic changes related to auditory hallucinations: a (31)P-MR spectroscopy study in antipsychotic-free schizophrenia patients. Brain Struct. Funct. 219, 1869-1872. doi: 10.1007/ s00429-013-0604-9

Nenadic, I., Smesny, S., Schlosser, R. G., Sauer, H., and Gaser, C. (2010). Auditory hallucinations and brain structure in schizophrenia: voxel-based morphometric study. Br. J. Psychiatry 196, 412-413. doi: 10.1192/bjp.bp.109.070441
Northoff, G., and Qin, P. (2011). How can the brain's resting state activity generate hallucinations? A 'resting state hypothesis' of auditory verbal hallucinations. Schizophr. Res. 127, 202-214. doi: 10.1016/j.schres.2010.11.009

O’Daly, O. G., Frangou, S., Chitnis, X., and Shergill, S. S. (2007). Brain structural changes in schizophrenia patients with persistent hallucinations. Psychiatry Res. 156, 15-21. doi: 10.1016/j.pscychresns.2007.03.001

O’Donnell, B. F., Vohs, J. L., Krishnan, G. P., Rass, O., Hetrick, W. P., and Morzorati, S. L. (2013). The auditory steady-state response (ASSR): a translational biomarker for schizophrenia. Suppl. Clin. Neurophysiol. 62, 101112. doi: 10.1016/b978-0-7020-5307-8.00006-5

Oestreich, L. K., McCarthy-Jones, S., and Whitford, T. J. (2016). Decreased integrity of the fronto-temporal fibers of the left inferior occipito-frontal fasciculus associated with auditory verbal hallucinations in schizophrenia. Brain Imaging Behav. 10, 445-454. doi: 10.1007/s11682-015-9421-5

Ohayon, S., Yitzhaky, A., and Hertzberg, L. (2020). Gene expression meta-analysis reveals the up-regulation of CREB1 and CREBBP in Brodmann Area 10 of patients with schizophrenia. Psychiatry Res. 292:113311. doi: 10.1016/j.psychres. 2020.113311

Palaniyappan, L., Balain, V., Radua, J., and Liddle, P. F. (2012). Structural correlates of auditory hallucinations in schizophrenia: a meta-analysis. Schizophr. Res. 137, 169-173. doi: 10.1016/j.schres.2012.01.038

Pergola, G., Di Carlo, P., Jaffe, A. E., Papalino, M., Chen, Q., Hyde, T. M., et al. (2019). Prefrontal coexpression of schizophrenia risk genes is associated with treatment response in patients. Biol. Psychiatry 86, 45-55. doi: 10.1016/j. biopsych.2019.03.981

Perrin, M. A., Kantrowitz, J. T., Silipo, G., Dias, E., Jabado, O., and Javitt, D. C. (2018). Mismatch negativity (MMN) to spatial deviants and behavioral spatial discrimination ability in the etiology of auditory verbal hallucinations and thought disorder in schizophrenia. Schizophr. Res. 191, 140-147. doi: 10.1016/j. schres.2017.05.012

Petralia, M. C., Ciurleo, R., Saraceno, A., Pennisi, M., Basile, M. S., Fagone, P., et al. (2020). Meta-Analysis of transcriptomic data of dorsolateral prefrontal cortex and of peripheral blood mononuclear cells identifies altered pathways in schizophrenia. Genes (Basel) 11:390. doi: 10.3390/genes11040390

Pinheiro, A. P., Farinha-Fernandes, A., Roberto, M. S., and Kotz, S. A. (2019). Selfvoice perception and its relationship with hallucination predisposition. Cogn. Neuropsychiatry 24, 237-255. doi: 10.1080/13546805.2019.1621159

Potter, D., Summerfelt, A., Gold, J., and Buchanan, R. W. (2006). Review of clinical correlates of P50 sensory gating abnormalities in patients with schizophrenia. Schizophr. Bull. 32, 692-700. doi: 10.1093/schbul/sbj050

Powers, A. R., Mathys, C., and Corlett, P. R. (2017). Pavlovian conditioninginduced hallucinations result from overweighting of perceptual priors. Science 357, 596-600. doi: 10.1126/science.aan3458

Psomiades, M., Fonteneau, C., Mondino, M., Luck, D., Haesebaert, F., SuaudChagny, M. F., et al. (2016). Integrity of the arcuate fasciculus in patients with schizophrenia with auditory verbal hallucinations: a DTI-tractography study. Neuroimage Clin. 12, 970-975. doi: 10.1016/j.nicl.2016.04.013

Rajasekaran, A., Shivakumar, V., Kalmady, S. V., Narayanaswamy, J. C., Subbana, M., Venugopal, D., et al. (2016). The impact of HLA-G 3' UTR variants and sHLA-G on risk and clinical correlates of schizophrenia. Hum. Immunol. 77, 1166-1171. doi: 10.1016/j.humimm.2016.08.013

Rivero, O., Sanjuan, J., Aguilar, E. J., Gonzalez, J. C., Molto, M. D., de Frutos, R., et al. (2010). Serotonin transporter gene polymorphisms and auditory hallucinations in psychosis. Rev. Neurol. 50, 325-332. doi: 10.33588/rn.5006. 2009387

Rolland, B., Amad, A., Poulet, E., Bordet, R., Vignaud, A., Bation, R., et al. (2015). Resting-state functional connectivity of the nucleus accumbens in auditory and visual hallucinations in schizophrenia. Schizophr. Bull. 41, 291-299. doi: $10.1093 /$ schbul/sbu097

Rotarska-Jagiela, A., Oertel-Knoechel, V., DeMartino, F., van de Ven, V., Formisano, E., Roebroeck, A., et al. (2009). Anatomical brain connectivity and positive symptoms of schizophrenia: a diffusion tensor imaging study. Psychiatry Res. 174, 9-16. doi: 10.1016/j.pscychresns.2009.03.002

Rudolph, E. D., Ells, E. M., Campbell, D. J., Abriel, S. C., Tibbo, P. G., Salisbury, D. F., et al. (2015). Finding the missing-stimulus mismatch negativity (MMN) in early psychosis: altered MMN to violations of an auditory gestalt. Schizophr. Res. 166, 158-163. doi: 10.1016/j.schres.2015.05.028 
Salisbury, D. F. (2012). Finding the missing stimulus mismatch negativity (MMN): emitted MMN to violations of an auditory gestalt. Psychophysiology 49, 544548. doi: 10.1111/j.1469-8986.2011.01336.x

Sanjuán, J., Castro-Martínez, X. H., García-Martí, G., González-Fernández, J., Sanz-Requena, R., Haro, J. M., et al. (2021). FOXP2 expression and gray matter density in the male brains of patients with schizophrenia. Brain Imaging Behav. 15, 1403-1411. doi: 10.1007/s11682-020-00339-x

Sanjuan, J., Rivero, O., Aguilar, E. J., Gonzalez, J. C., Molto, M. D., de Frutos, R., et al. (2006a). Serotonin transporter gene polymorphism (5-HTTLPR) and emotional response to auditory hallucinations in schizophrenia. Int. J. Neuropsychopharmacol. 9, 131-133. doi: 10.1017/s1461145705005559

Sanjuan, J., Toirac, I., Gonzalez, J. C., Leal, C., Molto, M. D., Najera, C., et al. (2004). A possible association between the CCK-AR gene and persistent auditory hallucinations in schizophrenia. Eur. Psychiatry 19, 349-353.

Sanjuan, J., Tolosa, A., Gonzalez, J. C., Aguilar, E. J., Perez-Tur, J., Najera, C., et al. (2006b). Association between FOXP2 polymorphisms and schizophrenia with auditory hallucinations. Psychiatr. Genet. 16, 67-72. doi: 10.1097/01.ypg. 0000185029.35558.bb

Scheinost, D., Tokoglu, F., Hampson, M., Hoffman, R., and Constable, R. T. (2019). Data-Driven analysis of functional connectivity reveals a potential auditory verbal hallucination network. Schizophr. Bull. 45, 415-424. doi: 10.1093/schbul/ sby039

Seok, J. H., Park, H. J., Chun, J. W., Lee, S. K., Cho, H. S., Kwon, J. S., et al. (2007). White matter abnormalities associated with auditory hallucinations in schizophrenia: a combined study of voxel-based analyses of diffusion tensor imaging and structural magnetic resonance imaging. Psychiatry Res. 156, 93104. doi: $10.1016 /$ j.pscychresns.2007.02.002

Shapleske, J., Rossell, S. L., Chitnis, X. A., Suckling, J., Simmons, A., Bullmore, E. T., et al. (2002). A computational morphometric MRI study of schizophrenia: effects of hallucinations. Cereb. Cortex 12, 1331-1341. doi: 10.1093/cercor/12. 12.1331

Shergill, S. S., Brammer, M. J., Williams, S. C., Murray, R. M., and McGuire, P. K. (2000). Mapping auditory hallucinations in schizophrenia using functional magnetic resonance imaging. Arch. Gen. Psychiatry 57, 1033-1038. doi: 10.1001/ archpsyc.57.11.1033

Shergill, S. S., Kanaan, R. A., Chitnis, X. A., O’Daly, O., Jones, D. K., Frangou, S., et al. (2007). A diffusion tensor imaging study of fasciculi in schizophrenia. Am. J. Psychiatry 164, 467-473. doi: 10.1176/ajp.2007.164.3.467

Shin, S. E., Lee, J. S., Kang, M. H., Kim, C. E., Bae, J. N., and Jung, G. (2005). Segmented volumes of cerebrum and cerebellum in first episode schizophrenia with auditory hallucinations. Psychiatry Res. 138, 33-42. doi: 10.1016/j.pscychresns.2004.11.005

Shinn, A. K., Baker, J. T., Cohen, B. M., and Ongur, D. (2013). Functional connectivity of left Heschl's gyrus in vulnerability to auditory hallucinations in schizophrenia. Schizophr. Res. 143, 260-268. doi: 10.1016/j.schres.2012. 11.037

Smith, D. M., Grant, B., Fisher, D. J., Borracci, G., Labelle, A., and Knott, V. J. (2013). Auditory verbal hallucinations in schizophrenia correlate with P50 gating. Clin. Neurophysiol. 124, 1329-1335. doi: 10.1016/j.clinph.2013.02.004

Spalletta, G., Piras, F., Gravina, P., Bello, M. L., Bernardini, S., and Caltagirone, C. (2012). Glutathione S-transferase alpha 1 risk polymorphism and increased bilateral thalamus mean diffusivity in schizophrenia. Psychiatry Res. 203, 180183. doi: 10.1016/j.pscychresns.2011.12.016

Spencer, K. M., Niznikiewicz, M. A., Nestor, P. G., Shenton, M. E., and McCarley, R. W. (2009). Left auditory cortex gamma synchronization and auditory hallucination symptoms in schizophrenia. BMC Neurosci. 10:85. doi: 10.1186/ 1471-2202-10-85

Sritharan, A., Line, P., Sergejew, A., Silberstein, R., Egan, G., and Copolov, D. (2005). EEG coherence measures during auditory hallucinations in schizophrenia. Psychiatry Res. 136, 189-200. doi: 10.1016/j.psychres.2005.05. 010

Stephan, K. E., Friston, K. J., and Frith, C. D. (2009). Dysconnection in schizophrenia: from abnormal synaptic plasticity to failures of self-monitoring. Schizophr. Bull. 35, 509-527. doi: 10.1093/schbul/sbn176

Tachikawa, H., Harada, S., Kawanishi, Y., Okubo, T., and Suzuki, T. (2001). Linked polymorphisms $(-333 \mathrm{G}>\mathrm{T}$ and $-286 \mathrm{~A}>\mathrm{G})$ in the promoter region of the CCKA receptor gene may be associated with schizophrenia. Psychiatry Res. 103, 147-155.
Talbot, K., Eidem, W. L., Tinsley, C. L., Benson, M. A., Thompson, E. W., Smith, R. J., et al. (2004). Dysbindin-1 is reduced in intrinsic, glutamatergic terminals of the hippocampal formation in schizophrenia. J. Clin. Invest. 113, 1353-1363. doi: $10.1172 /$ jci20425

Tang, J., LeGros, R. P., Louneva, N., Yeh, L., Cohen, J. W., Hahn, C.-G., et al. (2009). Dysbindin-1 in dorsolateral prefrontal cortex of schizophrenia cases is reduced in an isoform-specific manner unrelated to dysbindin-1 mRNA expression. Hum. Mol. Genet. 18, 3851-3863. doi: 10.1093/hmg/ddp329

Tang, T. T.-T., Yang, F., Chen, B.-S., Lu, Y., Ji, Y., Roche, K. W., et al. (2009). Dysbindin regulates hippocampal LTP by controlling NMDA receptor surface expression. Proc. Natl. Acad. Sci. U.S.A. 106, 21395-21400. doi: 10.1073/pnas. 0910499106

Tao, R., Li, C., Jaffe, A. E., Shin, J. H., Deep-Soboslay, A., Yamin, R., et al. (2020). Cannabinoid receptor CNR1 expression and DNA methylation in human prefrontal cortex, hippocampus and caudate in brain development and schizophrenia. Transl. Psychiatry 10:158. doi: 10.1038/s41398-020-0832-8

Theberge, J., Al-Semaan, Y., Williamson, P. C., Menon, R. S., Neufeld, R. W., Rajakumar, N., et al. (2003). Glutamate and glutamine in the anterior cingulate and thalamus of medicated patients with chronic schizophrenia and healthy comparison subjects measured with 4.0-T proton MRS. Am. J. Psychiatry 160, 2231-2233. doi: 10.1176/appi.ajp.160.12.2231

Thoma, R. J., Chaze, C., Lewine, J. D., Calhoun, V. D., Clark, V. P., Bustillo, J., et al. (2016). Functional MRI evaluation of multiple neural networks underlying auditory verbal hallucinations in schizophrenia spectrum disorders. Front. Psychiatry 7:39. doi: 10.3389/fpsyt.2016.00039

Thoma, R. J., Meier, A., Houck, J., Clark, V. P., Lewine, J. D., Turner, J., et al. (2017). Diminished auditory sensory gating during active auditory verbal hallucinations. Schizophr. Res. 188, 125-131. doi: 10.1016/j.schres.2017.01.023

Toirac, I., Sanjuan, J., Aguilar, E. J., Gonzalez, J. C., Artigas, F., Rivero, O., et al. (2007). Association between CCK-AR gene and schizophrenia with auditory hallucinations. Psychiatr. Genet. 17, 47-53.

Tolosa, A., Sanjuan, J., Dagnall, A. M., Molto, M. D., Herrero, N., and de Frutos, R. (2010). FOXP2 gene and language impairment in schizophrenia: association and epigenetic studies. BMC Med. Genet. 11:114. doi: 10.1186/1471-2350-11114

Tracy, D. K., and Shergill, S. S. (2013). Mechanisms underlying auditory hallucinations-understanding perception without stimulus. Brain Sci. 3, 642669. doi: 10.3390/brainsci3020642

Uhlhaas, P. J., Haenschel, C., Nikoliæ, D., and Singer, W. (2008). The role of oscillations and synchrony in cortical networks and their putative relevance for the pathophysiology of schizophrenia. Schizophr. Bull. 34, 927-943. doi: $10.1093 /$ schbul/sbn062

Uhrig, S., Hirth, N., Broccoli, L., von Wilmsdorff, M., Bauer, M., Sommer, C., et al. (2016). Reduced oxytocin receptor gene expression and binding sites in different brain regions in schizophrenia: a post-mortem study. Schizophr. Res. 177, 59-66. doi: 10.1016/j.schres.2016.04.019

van Lutterveld, R., Diederen, K. M., Koops, S., Begemann, M. J., and Sommer, I. E. (2013). The influence of stimulus detection on activation patterns during auditory hallucinations. Schizophr. Res. 145, 27-32. doi: 10.1016/j.schres.2013. 01.004

van Swam, C., Federspiel, A., Hubl, D., Wiest, R., Boesch, C., Vermathen, P., et al. (2012). Possible dysregulation of cortical plasticity in auditory verbal hallucinations-A cortical thickness study in schizophrenia. J. Psychiatr Res. 46, 1015-1023. doi: 10.1016/j.jpsychires.2012.03.016

Vawter, M. P., Hamzeh, A. R., Muradyan, E., Civelli, O., Abbott, G. W., and Alachkar, A. (2019). Association of myoinositol transporters with schizophrenia and bipolar disorder: evidence from human and animal studies. Mol. Neuropsychiatry 5, 200-211. doi: 10.1159/000501125

Vercammen, A., Knegtering, H., Bruggeman, R., and Aleman, A. (2011). Subjective loudness and reality of auditory verbal hallucinations and activation of the inner speech processing network. Schizophr. Bull. 37, 1009-1016. doi: 10.1093/schbul/ sbq007

Vercammen, A., Knegtering, H., den Boer, J. A., Liemburg, E. J., and Aleman, A. (2010). Auditory hallucinations in schizophrenia are associated with reduced functional connectivity of the temporo-parietal area. Biol. Psychiatry 67, 912918. doi: 10.1016/j.biopsych.2009.11.017

Waters, F., Collerton, D., Ffytche, D. H., Jardri, R., Pins, D., Dudley, R., et al. (2014). Visual hallucinations in the psychosis spectrum and comparative information 
from neurodegenerative disorders and eye disease. Schizophr. Bull. 40(Suppl. 4), S233-S245. doi: 10.1093/schbul/sbu036

Wei, J., and Hemmings, G. P. (1999). The CCK-A receptor gene possibly associated with auditory hallucinations in schizophrenia. Eur. Psychiatry 14, 67-70.

Weickert, C. S., Straub, R. E., McClintock, B. W., Matsumoto, M., Hashimoto, R., Hyde, T. M., et al. (2004). Human dysbindin (DTNBP1) gene expression in normal brain and in schizophrenic prefrontal cortex and midbrain. Arch. Gen. Psychiatry 61, 544-555. doi: 10.1001/archpsyc.61.6.544

Wigand, M., Kubicki, M., Clemm von Hohenberg, C., Leicht, G., Karch, S., Eckbo, R., et al. (2015). Auditory verbal hallucinations and the interhemispheric auditory pathway in chronic schizophrenia. World J. Biol. Psychiatry 16, 31-44. doi: 10.3109/15622975.2014.948063

Wolf, N. D., Gron, G., Sambataro, F., Vasic, N., Frasch, K., Schmid, M., et al. (2012). Magnetic resonance perfusion imaging of auditory verbal hallucinations in patients with schizophrenia. Schizophr. Res. 134, 285-287. doi: 10.1016/j. schres.2011.11.018

Wolf, N. D., Sambataro, F., Vasic, N., Frasch, K., Schmid, M., SchonfeldtLecuona, C., et al. (2011). Dysconnectivity of multiple resting-state networks in patients with schizophrenia who have persistent auditory verbal hallucinations. J. Psychiatry Neurosci. 36, 366-374. doi: 10.1503/jpn.110008

Wolpert, D. M., and Miall, R. C. (1996). Forward models for physiological motor control. Neural Netw. 9, 1265-1279. doi: 10.1016/s0893-6080(96)00035-4

Xi, Y. B., Guo, F., Li, H., Chang, X., Sun, J. B., Zhu, Y. Q., et al. (2016). The structural connectivity pathology of first-episode schizophrenia based on the cardinal symptom of auditory verbal hallucinations. Psychiatry Res. Neuroimaging 257, 25-30. doi: 10.1016/j.pscychresns.2016.09.011

Xie, S., Liu, B., Wang, J., Zhou, Y., Cui, Y., Song, M., et al. (2019). Hyperconnectivity in perisylvian language pathways in schizophrenia with auditory verbal hallucinations: a multi-site diffusion MRI study. Schizophr. Res. 210, 262-269. doi: 10.1016/j.schres.2018.12.024

Xu, J., Wang, J., Fan, L., Li, H., Zhang, W., Hu, Q., et al. (2015). Tractographybased parcellation of the human middle temporal gyrus. Sci. Rep. 5:18883. doi: $10.1038 /$ srep 18883

Youn, T., Park, H. J., Kim, J. J., Kim, M. S., and Kwon, J. S. (2003). Altered hemispheric asymmetry and positive symptoms in schizophrenia: equivalent current dipole of auditory mismatch negativity. Schizophr. Res. 59, 253-260. doi: 10.1016/s0920-9964(02)00154-8

Yu, H., Ying, W., Li, G., Lin, X., Jiang, D., Chen, G., et al. (2020). Exploring concomitant neuroimaging and genetic alterations in patients with and patients without auditory verbal hallucinations: a pilot study and mini review. J. Int. Med. Res. 48:300060519884856. doi: 10.1177/0300060519884856
Zhang, L., Li, B., Wang, H., Li, L., Liao, Q., Liu, Y., et al. (2017). Decreased middle temporal gyrus connectivity in the language network in schizophrenia patients with auditory verbal hallucinations. Neurosci. Lett. 653, 177-182. doi: 10.1016/j.neulet.2017.05.042

Zhao, Z., Li, X., Feng, G., Shen, Z., Li, S., Xu, Y., et al. (2018). Altered effective connectivity in the default network of the brains of first-episode, drugnaive schizophrenia patients with auditory verbal hallucinations. Front. Hum. Neurosci. 12:456. doi: 10.3389/fnhum.2018.00456

Zhu, J., Liu, C., Xiaodong Lin, M., Wang, L., Tian, H., and Chen, Q. (2019). Association of a novel long non-coding RNA-mRNAs network with default mode network hyper-connectivity in hallucinative schizophrenia. Ann. Clin. Case Rep. 2019:1657.

Zhuo, C., Li, G., Ji, F., Chen, C., Jiang, D., Lin, X., et al. (2020). Differences in functional connectivity density among subtypes of schizophrenic auditory hallucination. Brain Imaging Behav. 14, 2587-2593. doi: 10.1007/s11682-01900210-8

Zhuo, C., Zhu, J., Qin, W., Qu, H., Ma, X., and Yu, C. (2017). Cerebral blood flow alterations specific to auditory verbal hallucinations in schizophrenia. Br. J. Psychiatry 210, 209-215. doi: 10.1192/bjp.bp.115.17 4961

Zmigrod, L., Garrison, J. R., Carr, J., and Simons, J. S. (2016). The neural mechanisms of hallucinations: a quantitative meta-analysis of neuroimaging studies. Neurosci. Biobehav. Rev. 69, 113-123. doi: 10.1016/j.neubiorev.2016. 05.037

Conflict of Interest: The authors declare that the research was conducted in the absence of any commercial or financial relationships that could be construed as a potential conflict of interest.

Publisher's Note: All claims expressed in this article are solely those of the authors and do not necessarily represent those of their affiliated organizations, or those of the publisher, the editors and the reviewers. Any product that may be evaluated in this article, or claim that may be made by its manufacturer, is not guaranteed or endorsed by the publisher.

Copyright (c) 2021 Shao, Liao, Gu, Chen and Tang. This is an open-access article distributed under the terms of the Creative Commons Attribution License (CC BY). The use, distribution or reproduction in other forums is permitted, provided the original author(s) and the copyright owner(s) are credited and that the original publication in this journal is cited, in accordance with accepted academic practice. No use, distribution or reproduction is permitted which does not comply with these terms. 